\title{
On Disturbance Rejection for a Class of Nonlinear Systems
}

\author{
Wei Wei \\ School of Computer and Information Engineering, Beijing Key Laboratory of Big Data Technology for Food Safety, \\ Beijing Technology and Business University, Beijing 100048, China \\ Correspondence should be addressed to Wei Wei; weiweizdh@126.com
}

Received 17 February 2018; Revised 18 April 2018; Accepted 29 April 2018; Published 5 August 2018

Academic Editor: Jing $\mathrm{Na}$

Copyright (C) 2018 Wei Wei. This is an open access article distributed under the Creative Commons Attribution License, which permits unrestricted use, distribution, and reproduction in any medium, provided the original work is properly cited.

System control techniques have been developing for a long time. For advanced system requirements, sophisticated control algorithms are necessary for the nonlinear systems with uncertainties and disturbances. Disturbance attenuation or rejection control has been attracting an increasing attention from both control theory researchers and control engineering practitioners. In this paper, a new disturbance rejection control is proposed. Controllable canonical form is taken as the standard form of system dynamics, and a disturbance observer is taken to estimate the discrepancy between system dynamics and its standard form. Then the discrepancy could be compensated by control laws. Conditions of the closed-loop stability and ultimate bound of the tracking error have been obtained. Numerical results have also been presented to support the proposed approach.

\section{Introduction}

Automatic control plays a critical role in most of the engineering fields. Automatic control technology, which is capable of realizing the desired objectives without interference of human beings, has been developing all the time. For complex processes and advanced system requirements, a sophisticated control approach, which is able to optimize system performance and deal with interactions, nonlinearities, operating constrains, time-delay, and uncertainties, is of great necessity. For the sake of improving system performance in the presence of various uncertainties and disturbances, numerous advanced control algorithms and intelligent control methods, such as adaptive control, robust control, sliding mode control, model predictive control, neural network control, fuzzy control, and evolutionary computing techniques, have been proposed. Štefan Kozák has made an overview for the development of control engineering methods and structures in [1].

Actually, interactions, nonlinearities, time delays, and uncertainties are ubiquitous in industrial processes. Those are key factors degrading system performance.
Therefore, practically, the control problem is how to deal with those undesired factors so as to keep system performance still be satisfied [2]. If we define those issues as disturbance, then disturbance is a critical factor to corrupt a nominal course of actions. From this point of view, disturbance rejection is the key target in control $[2,3]$.

If disturbance is available, feed-forward control is a natural and optimal choice to attenuate or reject disturbance. However, disturbance is difficult to be available in advance. Thus, estimating disturbance is an alternative and effective way to solve this problem. Based on the estimation of disturbance, a control algorithm can be designed to suppress or cancel disturbance. Consequently, the closed-loop system performance could be guaranteed.

Motivated by such idea, researchers and practitioners have proposed a wide variety of disturbance attenuation/ rejection control algorithms. Since the 1960s, numerous disturbance estimation techniques, that is, the core of disturbance attenuation/rejection control algorithms, have been reported, such as disturbance observer (DOB) technique in disturbance observer-based control (DOBC) [4-6], unknown input observer (UIO) technique in disturbance 
accommodation control (DAC) [7], extended-state observer (ESO) technique in active disturbance rejection control (ADRC) [8], perturbation observer (POB) technique [9], and generalized proportional integral observer (GPIO) technique [10]. A review on the reported disturbance estimation techniques can be found in $[3,11,12]$.

Among the reported techniques, $\mathrm{DOB}$ has been initiatively put forward by Ohishi et al. in the early 1980s to improve torque and speed control [13]. In DOBC, disturbance distinctly refers to something external [2], while, ESO, first proposed by Han in the 1990s [14], is developed to be the key part of ADRC. In ADRC, any discrepancy between the standard form (i.e., cascade of integrators) and system dynamics will be viewed as the generalized disturbances. Therefore, not only external disturbances but also internal unmodeled dynamics and unknown uncertainties are within the range of generalized disturbance.

Also, considering that physical processes may be subject to different types of disturbance, composite hierarchical antidisturbance control (CHADC) has been proposed to avoid the conservativeness of disturbance estimation and rejection in the presence of multiple disturbances $[15,16]$.

Now, disturbance attenuation/rejection control has become a hot topic in recent years $[2,3]$. Within such framework, a nonlinear or linear controller is designed based on a nominal or standard model in the absence of disturbances and uncertainties, and its main work is to stabilize the system and achieve desired tracking performance. Then a nonlinear or linear disturbance observer is designed to estimate external disturbances and/or internal uncertainties and unmodeled system dynamics. Since disturbance attenuation/rejection control approaches are effective in engineering, it is not surprising that a large number of applications could be found in various industrial sectors, such as mechatronics systems, chemical and process systems, and aerospace systems $[3,17-20]$.

In this paper, we also focus on the disturbance attenuation/rejection control. The major contribution of this paper is to develop a general framework of a new disturbance rejection control design approach. Unlike ADRC, controllable canonical form is taken as the standard system dynamics. Disturbance observer is utilized to estimate the disturbance, which is defined as the discrepancy between the controllable canonical form and practical system dynamics. Based on the disturbance estimation and compensation, the system is dynamically linearized. Poles of the closed-loop system and the state observer can be assigned by setting tunable parameters of the baseline controller and the state observer. Clear physical explanations of parameters are helpful for controller design and its tuning. The input-to-state stability and ultimate bound of tracking error are obtained for a class of uncertain nonlinear systems.

The paper is organized as follows. A class of nonlinear system with uncertainties is presented in Section 2. A new disturbance rejection control, including its closed-loop stability and the tracking error, is analyzed in Section 3. In Section 4, numerical simulations are performed to support the proposed algorithm, and then conclusions and outlooks are drawn in Section 5.

\section{Problem Statement}

Consider an $n$th order nonlinear dynamical system

$$
\begin{aligned}
\dot{x}_{i} & =x_{i+1}, \\
\dot{x}_{n} & =f(\mathbf{x}, t)+d(t)+u(t), \\
y & =x_{1},
\end{aligned}
$$

where $\mathbf{x}=\left[x_{1}, x_{2}, \ldots, x_{n}\right]^{T} \in \mathbf{R}^{n}, f(\mathbf{x}, t) \in \mathbf{R}, d(t) \in \mathbf{R}, u(t) \in \mathbf{R}$, and $y \in \mathbf{R} . f(\mathbf{x}, t)$ is an unknown differentiable nonlinear function, which represents internal uncertainties and unmodelled dynamics. $d(t)$ is the unknown differentiable external disturbance, $u(t)$ is the control input, and $y$ is the system output.

System control input $u(t)$ is designed to drive system output $y$ to track desired output $y_{r}$ in the presence of unknown dynamics and external disturbances.

If we let

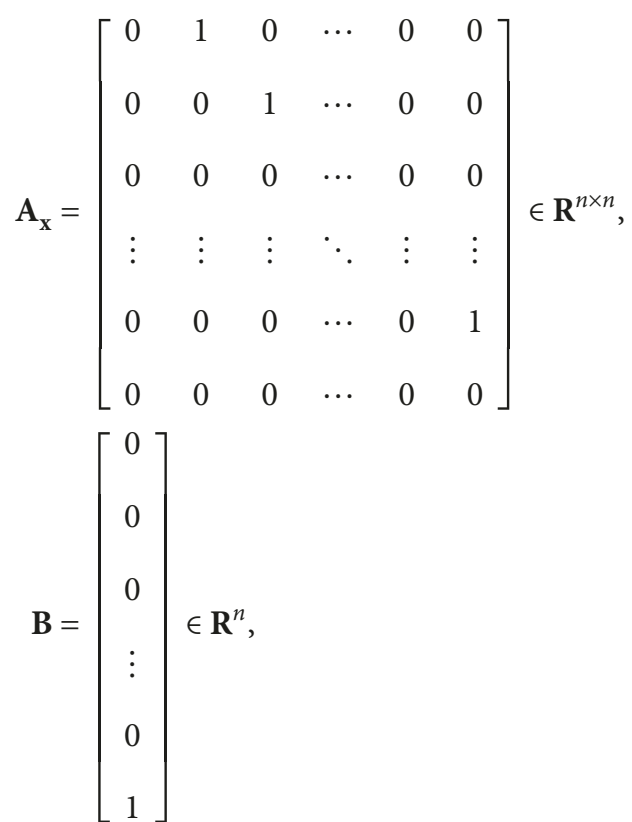

$$
\begin{aligned}
\mathbf{C}^{T} & =\left[\begin{array}{c}
1 \\
0 \\
0 \\
\vdots \\
0 \\
0
\end{array}\right] \in \mathbf{R}^{n}, \\
D & =f(\mathbf{x}, t)+d(t) \in \mathbf{R},
\end{aligned}
$$

system (1) can be rewritten as 


$$
\begin{aligned}
& \dot{\mathbf{x}}=\mathbf{A}_{\mathbf{x}} \mathbf{x}+\mathbf{B}(u+D), \\
& y=\mathbf{C} \mathbf{x} .
\end{aligned}
$$

Solving (3), we have

$$
y=\mathbf{C} \exp \left(\mathbf{A}_{\mathbf{x}} t\right) \mathbf{x}(0)+\mathbf{C} \int_{0}^{t} \exp \left(\mathbf{A}_{\mathbf{x}}(t-\tau)\right) \mathbf{B}(u+D)(\tau) d \tau .
$$

Obviously, $D$, that is, internal uncertainties, unmodelled dynamics, and unknown external disturbance, definitely affects system output. System output $y$ can be decoupled from $D$, if control input $u$ includes a part which is able to cancel $D$.

Let $u=u_{t r}+u_{D}$, where $u_{t r}$ is designed to stabilize the system and track the desired trajectory, and $u_{D}$ is designed to cancel $D$. Then we have

$$
\begin{gathered}
y=\mathbf{C}\left[\exp \left(\mathbf{A}_{\mathbf{x}} t\right) \mathbf{x}(0)+\int_{0}^{t} \exp \left(\mathbf{A}_{\mathbf{x}}(t-\tau)\right) \mathbf{B} u_{t r}(\tau) d \tau\right. \\
\left.+\int_{0}^{t} \exp \left(\mathbf{A}_{\mathbf{x}}(t-\tau)\right) \mathbf{B}\left(u_{D}+D\right)(\tau) d \tau\right] .
\end{gathered}
$$

Apparently, when $u_{D}+D=0$, system output will not be corrupted by $D$.

Hence, in this paper, we focus on the control algorithm, which is capable of cancelling uncertainties, unmodelled dynamics, and unknown external disturbances. A new disturbance rejection control approach is proposed for a class of nonlinear systems with uncertainties.

\section{Disturbance Rejection Control}

3.1. Disturbance Rejection Control Design. Disturbance rejection control law can be designed as

$$
\begin{aligned}
u & =u_{0}-\widehat{D}, \\
u_{0} & =-\mathbf{a}^{T} \widehat{\mathbf{x}}+\mathbf{a}^{T} \mathbf{y}_{r},
\end{aligned}
$$

where $u_{0}$ is the baseline controller, which is utilized to stabilize the system and track the desired trajectory, and $\widehat{D}$ is the disturbance observer, which is designed to estimate the unknown nonlinear dynamics $f(\mathbf{x}, t)$ and external disturbance $d(t)$, that is, $\widehat{D}=\hat{f}+\widehat{d}$. $u$ is the control signal. $\mathbf{a}=\left[a_{n}, a_{n-1}, \ldots, a_{1}\right]^{T} \in \mathbf{R}^{n}$ is the parameter vector, $\widehat{\mathbf{x}}=$ $\left[\widehat{x}_{1}, \widehat{x}_{2}, \ldots, \widehat{x}_{n}\right]^{T} \in \mathbf{R}^{n}$ is the estimation of the system state, and $\mathbf{y}_{r}=\left[y_{r}, \dot{y}_{r}, \ldots, y_{r}^{(n-1)}\right]^{T} \in \mathbf{R}^{n}$ is the vector composed of the desired output signal and its derivatives.

Substituting (6) into (1), we have closed-loop system

$$
\begin{aligned}
& \dot{\mathbf{x}}=\mathbf{A} \mathbf{x}+\mathbf{B} U, \\
& y=\mathbf{C} \mathbf{x},
\end{aligned}
$$

where $U=\mathbf{a}^{T} \tilde{\mathbf{x}}+\mathbf{a}^{T} \mathbf{y}_{r}+\tilde{D} \in \mathbf{R}, \quad \tilde{\mathbf{x}}=\mathbf{x}-\widehat{\mathbf{x}} \in \mathbf{R}^{n}, \quad \tilde{D} \triangleq D-\widehat{D}$ $\in \mathbf{R}$, and

$$
\begin{aligned}
\mathbf{A} & =\mathbf{A}_{\mathbf{x}}-\mathbf{B a}^{T} \\
& =\left[\begin{array}{cccccc}
0 & 1 & 0 & \cdots & 0 & 0 \\
0 & 0 & 1 & \cdots & 0 & 0 \\
0 & 0 & 0 & \cdots & 0 & 0 \\
\vdots & \vdots & \vdots & \ddots & \vdots & \vdots \\
0 & 0 & 0 & \cdots & 0 & 1 \\
-a_{n} & -a_{n-1} & -a_{n-2} & \cdots & -a_{2} & -a_{1}
\end{array}\right] \in \mathbf{R}^{n \times n} .
\end{aligned}
$$

Apparently, system (7) is of controllable canonical form. In other words, by disturbance rejection control law (6), uncertain nonlinear system (1) is dynamically linearized to a linear time-invariant (LTI) system, which has the controllable canonical form.

Here, the state observer for system (3) is designed as

$$
\begin{aligned}
& \dot{\hat{\mathbf{x}}}=\mathbf{A}_{\mathbf{x}} \widehat{\mathbf{x}}+\mathbf{B} u_{0}+\mathbf{L}(y-\widehat{y}), \\
& \widehat{y}=\mathbf{C} \widehat{\mathbf{x}},
\end{aligned}
$$

where $\quad \widehat{\mathbf{x}}=\left[\widehat{x}_{1}, \widehat{x}_{2}, \ldots, \widehat{x}_{n}\right]^{T} \in \mathbf{R}^{n}, \quad \mathbf{L}=\left[l_{1}, l_{2}, \ldots, l_{n}\right]^{T} \in \mathbf{R}^{n}$, $u_{0} \in \mathbf{R}$, and $\hat{y} \in \mathbf{R}$.

Subtracting (9) from (3), we have

$$
\dot{\tilde{\mathbf{x}}}=\left(\mathbf{A}_{\mathbf{x}}-\mathbf{L C}\right) \tilde{\mathbf{x}}+\mathbf{B} \tilde{D}
$$

where $\tilde{\mathbf{x}}=\mathbf{x}-\widehat{\mathbf{x}} \in \mathbf{R}^{n}$ and let $\mathbf{A}_{G}=\mathbf{A}_{\mathbf{x}}-\mathbf{L C}$.

For disturbance observer, it can be designed as [5]

$$
\begin{aligned}
& \widehat{D}=\xi+p(\widehat{\mathbf{x}}), \\
& \dot{\xi}=-l_{n+1} \xi-l_{n+1}[p(\widehat{\mathbf{x}})+u],
\end{aligned}
$$

where $\xi \in \mathbf{R}, l_{n+1} \in \mathbf{R}$, and $p(\widehat{\mathbf{x}}) \in \mathbf{R}$.

The derivative of $\tilde{D}$ is designed as $\dot{\tilde{D}} \triangleq \dot{D}-\dot{\hat{D}}$. In general, there is no prior information about the derivative of the disturbance $D$. It is reasonable to suppose that $\dot{D}=0$, which implies that the disturbance varies slowly relative to the observer dynamics. Hence,

$$
\dot{\tilde{D}} \triangleq-\dot{\tilde{D}}=-[\dot{\xi}+\dot{p}(\widehat{\mathbf{x}})]=l_{n+1} \xi+l_{n+1}[p(\widehat{\mathbf{x}})+u]-\dot{p}(\widehat{\mathbf{x}}) .
$$

If we choose $p(\widehat{\mathbf{x}})=l_{n+1} \widehat{x}_{n}$, then (12) can be rewritten as

$$
\dot{\tilde{D}}=l_{n+1} \widehat{D}+l_{n+1}\left(u-\dot{\hat{x}}_{n}\right) \text {. }
$$

Since $\dot{\hat{x}}_{n}=u_{0}+l_{n} \mathbf{C} \tilde{\mathbf{x}}$, we have

$$
\dot{\tilde{D}}=l_{n+1} \widehat{D}+l_{n+1}\left(u-u_{0}-l_{n} \mathbf{C} \tilde{\mathbf{x}}\right)=-l_{n+1} l_{n} \mathbf{C} \tilde{\mathbf{x}} .
$$




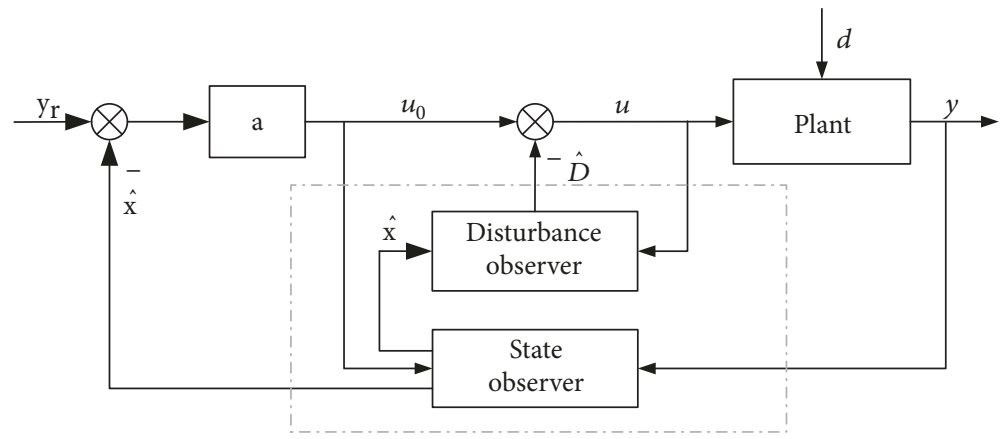

Figure 1: New disturbance rejection control diagram.

For the estimation error systems (10) and (14), we have

$$
\dot{\mathbf{z}}=\mathbf{A}_{\mathbf{z}} \mathbf{z}
$$

where

$$
\mathbf{z}=\left[\begin{array}{c}
\tilde{\mathbf{x}} \\
\tilde{D}
\end{array}\right] \in \mathbf{R}^{n+1}
$$

and

$$
\mathbf{A}_{\mathbf{z}}=\left[\begin{array}{cc}
\mathbf{A}_{G} & \mathbf{B} \\
-l_{n+1} l_{n} \mathbf{C} & 0
\end{array}\right] \in \mathbf{R}^{(n+1) \times(n+1)} .
$$

The solution of system (15) is $\mathbf{z}(t)=e^{\mathbf{A}_{\mathbf{z}} t} \mathbf{z}(0)$. Since $\mathbf{A}_{\mathbf{z}}$ is a finite constant matrix, if we choose proper $\left[\mathbf{L}: l_{n+1}\right]$ such that all eigenvalues of $\mathbf{A}_{\mathbf{z}}$ are negative, we have $\|\mathbf{z}(t)\| \leq \mathbf{z}(0)=\delta_{\mathbf{z}}$, that is,

$$
\left\|\left[\begin{array}{c}
\tilde{\mathbf{x}} \\
\tilde{D}
\end{array}\right]\right\| \leq \delta_{\mathbf{z}}
$$

Control block diagram is shown in Figure 1.

Next, the definition of input-to-state stability is given, and then the stability analysis has been presented.

Definition [21]. The system is said to be input-to-state stable if there exist a class $\mathbf{K L}$ function $\beta$ and a class $\mathbf{K}$ function $\gamma$ such that for any initial state $x\left(t_{0}\right)$ and any bounded input $u(t)$, the solution $x(t)$ exists for all $t \geq t_{0}$ and satisfies $\|x(t)\| \leq \beta\left(\left\|x\left(t_{0}\right)\right\|, t-t_{0}\right)+\gamma\left(\sup _{t_{0} \leq \tau \leq t}\|u(\tau)\|\right)$.

Accordingly, we have Theorem 1.

Theorem 1. Closed-loop system (7) is input-to-state stable, if we choose a proper parameter vector $\mathbf{a}$ and $\left[\mathbf{L} \vdots l_{n+1}\right]$, such that system matrix A is Hurwitz and estimation error is bounded.

Proof. For closed-loop system (7), its solution can be written as

$$
\mathbf{x}=\exp (\mathbf{A} t) \mathbf{x}(0)+\int_{0}^{t} \exp (\mathbf{A}(t-\tau)) \mathbf{B} U(\tau) d \tau
$$

Coefficients of characteristic polynomial are determined by a, that is, $|\lambda \mathbf{I}-\mathbf{A}|=\lambda^{n}+a_{1} \lambda^{n-1}+\cdots+a_{n-1} \lambda+a_{n}$.
(Here, $\mathbf{I}$ is the unit matrix.) If $\mathbf{a}$ is chosen properly, system matrix $\mathbf{A}$ will be Hurwitz. Let eigenvalues of system matrix A be $-\lambda_{i}, i=1,2, \ldots, n$. There exists $\kappa>0$, such that $\forall i$, $\operatorname{Re}\left[-\lambda_{i}\right]<-\kappa$, then $\|\exp (\mathbf{A} t)\| \leq M \exp (-\kappa t)$. Therefore, we have

$$
\begin{aligned}
\|\mathbf{x}\| & \leq M \exp (-\kappa t)\|\mathbf{x}(0)\|+\int_{0}^{t} M \exp (-\kappa(t-\tau))\|\mathbf{B}\|\|U(\tau)\| d \tau \\
& \leq M \exp (-\kappa t)\|\mathbf{x}(0)\|+\frac{M\|\mathbf{B}\|}{\kappa} \sup _{0 \leq \tau \leq t}\|U(\tau)\| .
\end{aligned}
$$

It shows that zero-input response decays to zero exponentially and zero-state response is proportional to the bound of the input.

Considering that

$$
\left\|\left[\begin{array}{c}
\tilde{\mathbf{x}} \\
\tilde{D}
\end{array}\right]\right\| \leq \delta_{\mathbf{z}}
$$

(when $\left[\mathbf{L}: l_{n+1}\right]$ is properly chosen) and $\mathbf{y}_{r}$ is also bounded, we have $U=\mathbf{a}^{T} \tilde{\mathbf{x}}+\mathbf{a}^{T} \mathbf{y}_{r}+\tilde{D}$ which is a bounded input signal. According to the definition of input-to-state stable, we can conclude that closed-loop system (7) is input-to-state stable. q.e.d.

3.2. Tracking Error. For input-to-state stable system (7), let tracking error be $e=y_{r}-y$, we have

$$
\begin{aligned}
& e=e_{1}=y_{r}-x_{1}, \\
& e_{2}=\dot{y}_{r}-\dot{x}_{1}, \\
& e_{3}=\ddot{y}_{r}-\ddot{x}_{1}, \ldots, \\
& e_{n}=y_{r}^{(n-1)}-x_{1}^{(n-1)} .
\end{aligned}
$$

Accordingly,

$$
\begin{aligned}
\dot{e}_{1} & =e_{2}, \\
\dot{e}_{2} & =e_{3}, \\
, \ldots & \\
\dot{e}_{n-1} & =e_{n}, \\
\dot{e}_{n} & =y_{r}^{(n)}-x_{1}^{(n)} .
\end{aligned}
$$

According to system (7), we have 


$$
\begin{aligned}
\dot{e}_{1} & =e_{2}, \\
\dot{e}_{2} & =e_{3}, \\
, \ldots & \\
\dot{e}_{n-1} & =e_{n}, \\
\dot{e}_{n} & =y_{r}^{(n)}+\mathbf{a}^{T} \widehat{\mathbf{x}}-\mathbf{a}^{T} \mathbf{y}_{r}-\tilde{D} .
\end{aligned}
$$

The last equation of system (24) can be written as

$$
\begin{aligned}
\dot{e}_{n} & =y_{r}^{(n)}+\mathbf{a}^{T} \widehat{\mathbf{x}}-\mathbf{a}^{T} \mathbf{y}_{r}-\tilde{D} \\
& =y_{r}^{(n)}+a_{n} \widehat{x}_{1}+a_{n-1} \widehat{x}_{2}+\cdots+a_{1} \widehat{x}_{n}-a_{n} y_{r}-\cdots-a_{1} y_{r}^{(n-1)}-\tilde{D} .
\end{aligned}
$$

Since $e_{1}=y_{r}-x_{1}, \quad e_{2}=\dot{y}_{r}-\dot{x}_{1}, \quad e_{3}=\ddot{y}_{r}-\ddot{x}_{1}, \ldots$, and $e_{n}=y_{r}^{(n-1)}-x_{1}^{(n-1)}$, that is,

$$
\begin{aligned}
& e_{1}=y_{r}-x_{1}, \\
& e_{2}=\dot{y}_{r}-x_{2}, \\
& e_{3}=\ddot{y}_{r}-x_{3}, \ldots, \\
& e_{n}=y_{r}^{(n-1)}-x_{n},
\end{aligned}
$$

we have $x_{1}=y_{r}-e_{1}, x_{2}=\dot{y}_{r}-e_{2}, x_{3}=\ddot{y}_{r}-e_{3}, \ldots$, and $x_{n}=y_{r}^{(n-1)}-e_{n}$.

For $\tilde{x}_{1}=x_{1}-\widehat{x}_{1}, \tilde{x}_{2}=x_{2}-\widehat{x}_{2}, \ldots$, and $\tilde{x}_{n}=x_{n}-\hat{x}_{n}$, then

$$
\begin{aligned}
& \widehat{x}_{1}=x_{1}-\tilde{x}_{1}, \\
& \widehat{x}_{2}=x_{2}-\tilde{x}_{2}, \ldots, \\
& \widehat{x}_{n}=x_{n}-\tilde{x}_{n} .
\end{aligned}
$$

Thus,

$$
\begin{aligned}
& \widehat{x}_{1}=y_{r}-e_{1}-\tilde{x}_{1}, \\
& \widehat{x}_{2}=\dot{y}_{r}-e_{2}-\tilde{x}_{2}, \ldots, \\
& \widehat{x}_{n}=y_{r}^{(n-1)}-e_{n}-\tilde{x}_{n} .
\end{aligned}
$$

Substituting (28) into (25), we have

$$
\begin{aligned}
\dot{e}_{n}= & -a_{n} e_{1}-a_{n-1} e_{2}+\cdots-a_{1} e_{n}-a_{n} \tilde{x}_{1}-a_{n-1} \tilde{x}_{2} \\
& +\cdots-a_{1} \tilde{x}_{n}-\tilde{D}+y_{r}^{(n)} .
\end{aligned}
$$

If we define $\mathbf{e}=\left[e_{1}, e_{2}, \ldots, e_{n}\right]^{T} \in \mathbf{R}^{n}, \tilde{\mathbf{x}}=\left[\tilde{x}_{1}, \tilde{x}_{2}, \ldots, \tilde{x}_{n}\right]^{T}$ $\in \mathbf{R}^{n}$, then (29) can be rewritten as

$$
\dot{e}_{n}=-\mathbf{a}^{T} \mathbf{e}-\mathbf{a}^{T} \tilde{\mathbf{x}}+y_{r}^{(n)}-\tilde{D} .
$$

According to (24) and (30), we have

$$
\begin{aligned}
\dot{e}_{1} & =e_{2}, \\
\dot{e}_{2} & =e_{3}, \\
, \ldots & \\
\dot{e}_{n-1} & =e_{n}, \\
\dot{e}_{n} & =-\mathbf{a}^{T} \mathbf{e}-\mathbf{a}^{T} \tilde{\mathbf{x}}+y_{r}^{(n)}-\tilde{D} .
\end{aligned}
$$

Equation (31), that is, the closed-loop tracking error system, can be written in a compact form

$$
\dot{\mathbf{e}}=\mathbf{A e}+\boldsymbol{\varepsilon}(t),
$$

where $\boldsymbol{\varepsilon}(t)=\left[0,0, \ldots, 0,-\mathbf{a}^{T} \tilde{\mathbf{x}}+y_{r}^{(n)}-\tilde{D}\right]^{T} \in \mathbf{R}^{n}$,

$$
\mathbf{A}=\left[\begin{array}{cccccc}
0 & 1 & 0 & \cdots & 0 & 0 \\
0 & 0 & 1 & \cdots & 0 & 0 \\
0 & 0 & 0 & \cdots & 0 & 0 \\
\vdots & \vdots & \vdots & \ddots & \vdots & \vdots \\
0 & 0 & 0 & \cdots & 0 & 1 \\
-a_{n} & -a_{n-1} & -a_{n-2} & \cdots & -a_{2} & -a_{1}
\end{array}\right] \in \mathbf{R}^{n \times n} .
$$

Since

$$
\left\|\left[\begin{array}{c}
\tilde{\mathbf{x}} \\
\tilde{D}
\end{array}\right]\right\| \leq \delta_{\mathbf{z}}
$$

and $y_{r}^{(n)}$ are also bounded, without loss of generality, we can assume that $\|\boldsymbol{\varepsilon}(t)\| \leq \delta$, where $\delta$ is a constant.

Before giving out the bound of tracking error, the following lemma can be presented.

Lemma [21]. Let $\mathbf{D} \subset \mathbf{R}^{n}$ be a domain that contains the origin and $V:[0, \infty) \times \mathbf{D} \rightarrow \mathbf{R}$ be a continuously differentiable function such that

$$
\begin{aligned}
& \alpha_{1}(\|\mathbf{x}\|) \leq V(t, \mathbf{x}) \leq \alpha_{2}(\|\mathbf{x}\|) \\
& \frac{\partial V}{\partial t}+\frac{\partial V}{\partial \mathbf{x}} f(t, \mathbf{x}) \leq-W_{3}(\mathbf{x}), \quad \forall\|\mathbf{x}\| \geq \mu>0 .
\end{aligned}
$$

$\forall t \geq 0$ and $\forall \mathbf{x} \in \mathbf{D}$, where $\alpha_{1}$ and $\alpha_{2}$ are class $\boldsymbol{K}$ functions and $W_{3}(\mathbf{x})$ is a continuous positive definite function. Take $r>0$ such that $B_{r} \subset \mathbf{D}$ and suppose that

$$
\mu<\alpha_{2}^{-1}\left(\alpha_{1}(r)\right) \text {. }
$$

Then there exists a class $K L$ function $\beta$ for every initial state $\mathbf{x}\left(t_{0}\right)$, satisfying $\left\|\mathbf{x}\left(t_{0}\right)\right\| \leq \alpha_{2}^{-1}\left(\alpha_{1}(r)\right)$, and there is $T \geq 0$ (dependent on $\mathbf{x}\left(t_{0}\right)$ and $\mu$ ) such that the solution of $\dot{\mathbf{x}}=f(t, \mathbf{x})$ satisfies

$$
\begin{aligned}
& \|\mathbf{x}(t)\| \leq \beta\left(\left\|\mathbf{x}\left(t_{0}\right)\right\|, t-t_{0}\right), \quad \forall t_{0} \leq t \leq t_{0}+T, \\
& \|\mathbf{x}(t)\| \leq \alpha_{1}^{-1}\left(\alpha_{2}(\mu)\right), \quad \forall t \geq t_{0}+T .
\end{aligned}
$$

Moreover, if $\mathbf{D}=\mathbf{R}^{n}$ and $\alpha_{1}$ belongs to class $\boldsymbol{K}_{\infty}$, then (37) holds for any initial state $\mathbf{x}\left(t_{0}\right)$, with no restriction on how large $\mu$ is.

Then Theorem 2 can be obtained.

Theorem 2. For closed-loop tracking error system (32), if $\lambda_{\max }(\mathbf{A})<-\left(\delta /\|\mathbf{e}\|_{2}\right)$, the tracking error $\|\mathbf{e}\|=O(1)$, and the ultimate bound is $-\left(\delta /\left(\lambda_{\max }(\mathbf{A})\right) \sqrt{\left(\lambda_{\max }(\mathbf{P})\right) /\left(\lambda_{\min }(\mathbf{P})\right)}\right.$.

Proof. Note that, if parameters of controller (6) have been selected properly, negative eigenvalues of matrix $\mathbf{A}$ in system (32) is distinct. Considering that the system matrix $\mathbf{A}$ is of the controllable canonical form, it can be transformed to a 


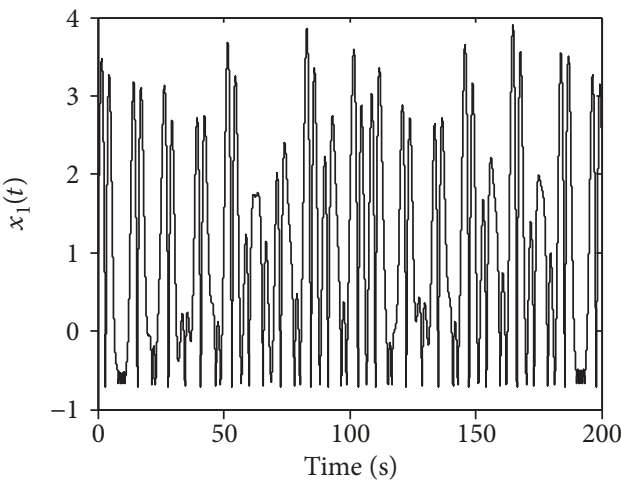

(a)

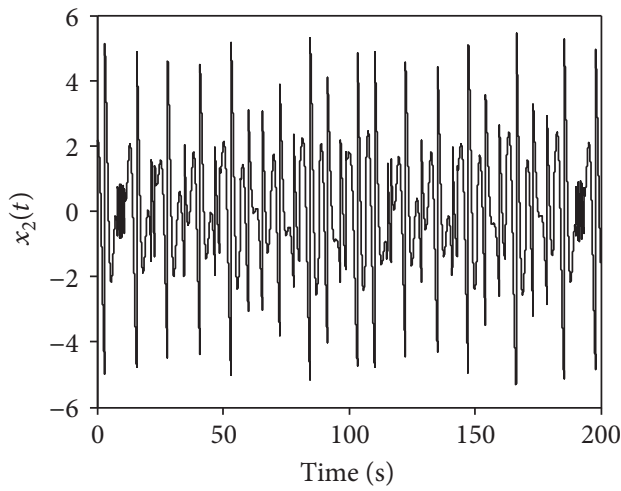

(b)

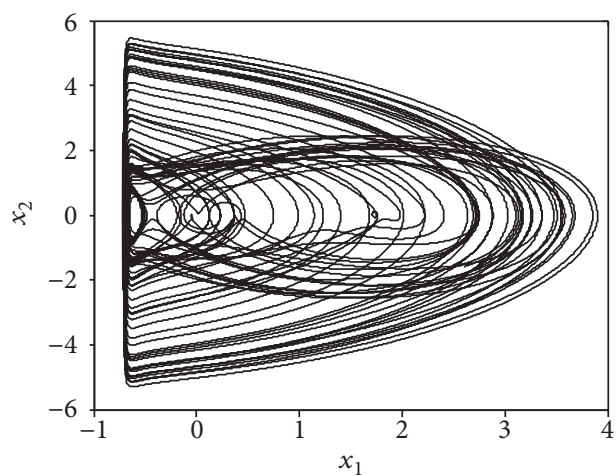

(c)

FIgURE 2: Chaotic dynamics and attractor of a microcantilever in the AFM system.

TABLE 1: Control parameters of NDRC and ADRC (i).

\begin{tabular}{lcccccccc}
\hline Controller & $\omega_{\mathrm{o}}$ & $l_{3} / \omega_{c}$ & $\lambda_{1} / b_{0}$ & $\lambda_{2} / \beta_{1}$ & $a_{1} / \beta_{2}$ & $a_{2} / \beta_{3}$ & $k_{1}$ & $k_{2}$ \\
\hline NDRC (i) & 120 & 36 & -8 & -9 & 17 & 72 & - & - \\
NDRC (ii) & 100 & 36 & -12 & -21 & 33 & 252 & - & - \\
ADRC & 75 & 15 & 1 & 225 & 16,875 & 421,875 & 30 & 225 \\
\hline
\end{tabular}

diagonal matrix by a Vandermonde matrix. The transformation matrix is

$$
\mathbf{T}=\left[\begin{array}{ccccc}
1 & 1 & 1 & \cdots & 1 \\
-\lambda_{1} & -\lambda_{2} & -\lambda_{3} & \cdots & -\lambda_{n} \\
\left(-\lambda_{1}\right)^{2} & \left(-\lambda_{2}\right)^{2} & \left(-\lambda_{3}\right)^{2} & \cdots & \left(-\lambda_{n}\right)^{2} \\
\vdots & \vdots & \vdots & \ddots & \vdots \\
\left(-\lambda_{1}\right)^{n-1} & \left(-\lambda_{2}\right)^{n-1} & \left(-\lambda_{3}\right)^{n-1} & \cdots & \left(-\lambda_{n}\right)^{n-1}
\end{array}\right]
$$

where $-\lambda_{1},-\lambda_{2}, \ldots,-\lambda_{n}$ are eigenvalues of matrix $\mathbf{A}$, and supposing that $\lambda_{n}>\cdots>\lambda_{2}>\lambda_{1}>0$, we have the nonsingular transformation $\mathbf{e}=\mathbf{T} \overline{\mathbf{e}}$, which transforms system (32) to be

$$
\dot{\overline{\mathbf{e}}}=\overline{\mathbf{A}} \overline{\mathbf{e}}+\mathbf{T}^{-1} \boldsymbol{\varepsilon}(t),
$$

where $\overline{\mathbf{A}}=\mathbf{T}^{-1} \mathbf{A} \mathbf{T}=-\operatorname{diag}\left[\lambda_{1}, \lambda_{2}, \ldots, \lambda_{n}\right]$.
Let $\mathbf{P}=\left(\mathbf{T}^{-1}\right)^{T}\left(\mathbf{T}^{-1}\right)$, we can define a Lyapunov function candidate as

$$
\begin{aligned}
V(\mathbf{e}) & =\frac{1}{2} \mathbf{e}^{T} \mathbf{P e}=\frac{1}{2}(\mathbf{T} \overline{\mathbf{e}})^{T} \mathbf{P}(\mathbf{T} \overline{\mathbf{e}})=\frac{1}{2} \overline{\mathbf{e}}^{T} \mathbf{T}^{T} \mathbf{P} \mathbf{T} \overline{\mathbf{e}} \\
& =\frac{1}{2} \overline{\mathbf{e}}^{T} \mathbf{T}^{T}\left(\mathbf{T}^{-1}\right)^{T}\left(\mathbf{T}^{-1}\right) \mathbf{T} \overline{\mathbf{e}}=\frac{1}{2} \overline{\mathbf{e}}^{T} \mathbf{T}^{T}\left(\mathbf{T}^{T}\right)^{-1}\left(\mathbf{T}^{-1}\right) \mathbf{T} \overline{\mathbf{e}} \\
& =\frac{1}{2} \overline{\mathbf{e}}^{T} \overline{\mathbf{e}},
\end{aligned}
$$

then we have the derivative of $V(\mathbf{e})$ along system (39),

$$
\begin{aligned}
\dot{V}(\mathbf{e}) & =\frac{1}{2}\left(\dot{\overline{\mathbf{e}}}^{T} \overline{\mathbf{e}}+\overline{\mathbf{e}}^{T} \dot{\overline{\mathbf{e}}}\right)=\frac{1}{2}\left[\overline{\mathbf{A}} \overline{\mathbf{e}}+\mathbf{T}^{-1} \boldsymbol{\varepsilon}(t)\right]^{T} \overline{\mathbf{e}}+\frac{1}{2} \overline{\mathbf{e}}^{T}\left[\overline{\mathbf{A}} \overline{\mathbf{e}}+\mathbf{T}^{-1} \boldsymbol{\varepsilon}(t)\right] \\
& =\frac{1}{2}\left[\overline{\mathbf{e}}^{T} \overline{\mathbf{A}}^{T}+\boldsymbol{\varepsilon}^{T}\left(\mathbf{T}^{-1}\right)^{T}\right]^{T} \overline{\mathbf{e}}+\frac{1}{2} \overline{\mathbf{e}}^{T}\left[\overline{\mathbf{A}} \overline{\mathbf{e}}+\mathbf{T}^{-1} \boldsymbol{\varepsilon}\right] \\
& =\frac{1}{2}\left[\overline{\mathbf{e}}^{T} \overline{\mathbf{A}}^{T} \overline{\mathbf{e}}+\boldsymbol{\varepsilon}^{T}\left(\mathbf{T}^{-1}\right)^{T} \overline{\mathbf{e}}+\overline{\mathbf{e}}^{T} \overline{\mathbf{A}} \overline{\mathbf{e}}+\overline{\mathbf{e}}^{T} \mathbf{T}^{-1} \boldsymbol{\varepsilon}\right] \\
& =\frac{1}{2}\left[2 \overline{\mathbf{e}}^{T} \overline{\mathbf{A}} \overline{\mathbf{e}}+\left[\overline{\mathbf{e}}^{T} \mathbf{T}^{-1} \boldsymbol{\varepsilon}\right]^{T}+\overline{\mathbf{e}}^{T} \mathbf{T}^{-1} \boldsymbol{\varepsilon}\right]=\overline{\mathbf{e}}^{T} \overline{\mathbf{A}} \overline{\mathbf{e}}+\overline{\mathbf{e}}^{T} \mathbf{T}^{-1} \boldsymbol{\varepsilon}, \\
\dot{V}(\mathbf{e}) & =\overline{\mathbf{e}}^{T} \overline{\mathbf{A}} \overline{\mathbf{e}}+\overline{\mathbf{e}}^{T} \mathbf{T}^{-1} \boldsymbol{\varepsilon} \leq-\lambda_{1}\|\overline{\mathbf{e}}\|_{2}^{2}+\|\overline{\mathbf{e}}\|_{2}\left\|\mathbf{T}^{-1}\right\|_{2}\|\boldsymbol{\varepsilon}\|_{2} \\
& \leq-\left(\lambda_{1}-\frac{\delta}{\|\overline{\mathbf{e}}\|_{2}}\left\|\mathbf{T}^{-1}\right\|_{2}\right)\|\overline{\mathbf{e}}\|_{2}^{2} .
\end{aligned}
$$

If $\dot{V}(\mathbf{e})<0$, we have $\|\overline{\mathbf{e}}\|_{2}>\mu, \mu=\left(\delta / \lambda_{1}\right)\left\|\mathbf{T}^{-1}\right\|_{2}$. 


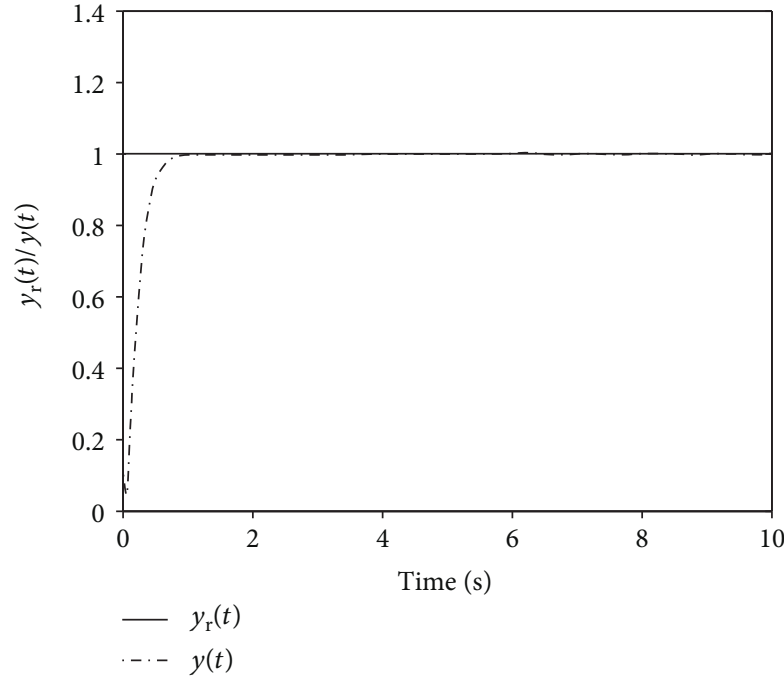

(a)

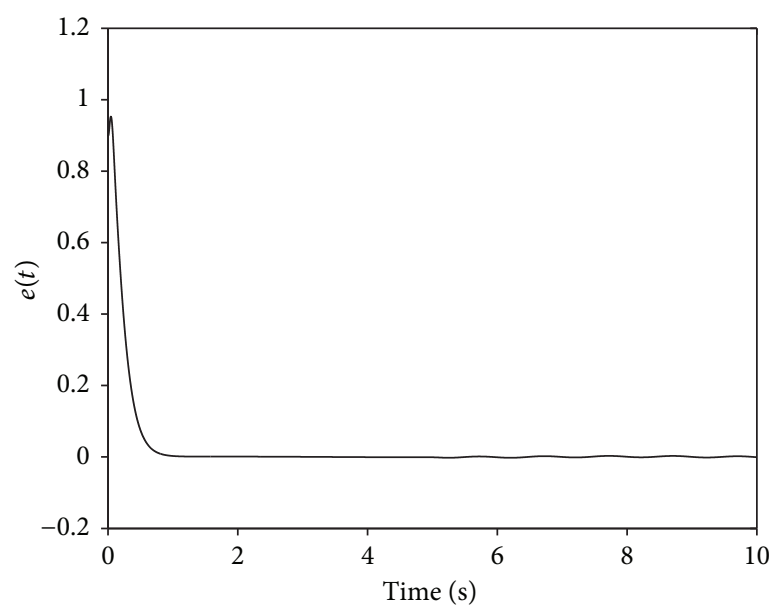

(c)

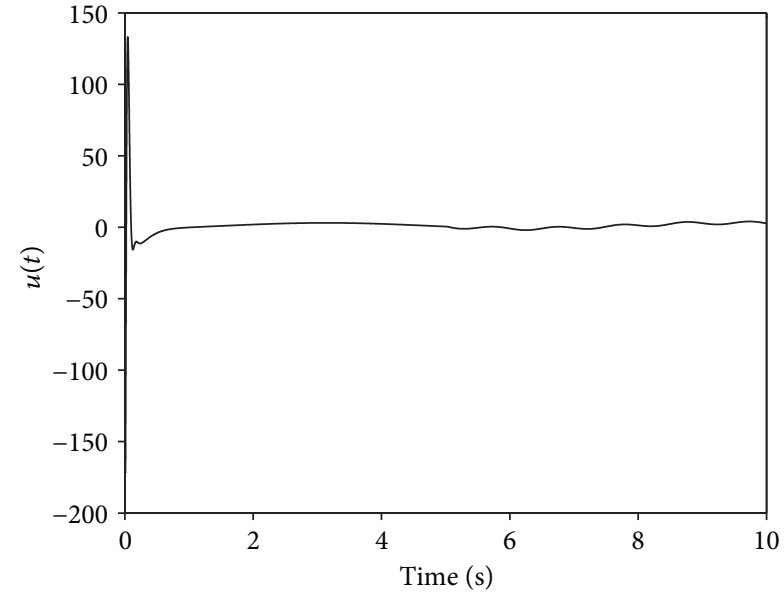

(b)

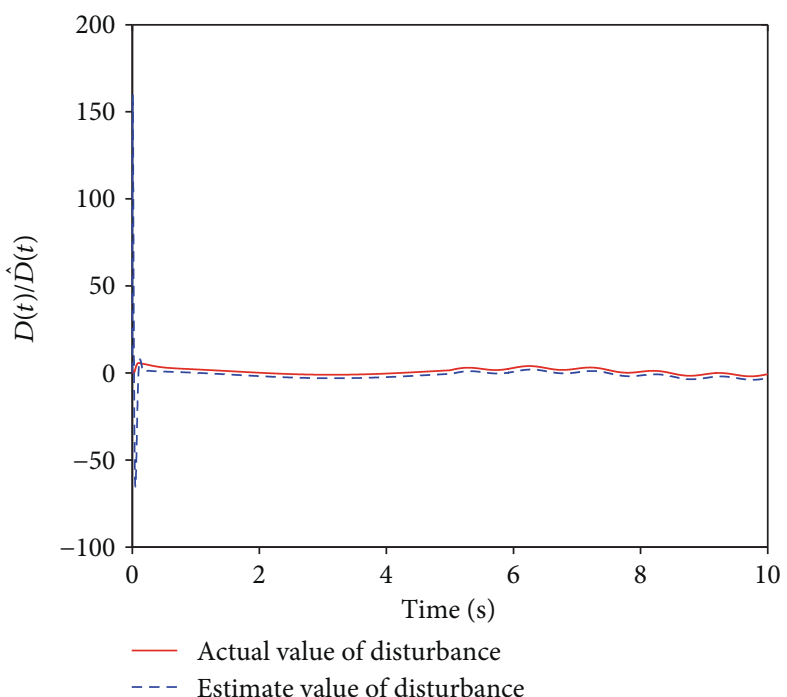

(d)

FIgURE 3: System response of a microcantilever in AFM by NDRC.

For $\mathbf{e}=\mathbf{T} \overline{\mathbf{e}}$, we have $\overline{\mathbf{e}}=\mathbf{T}^{-1} \mathbf{e}$; then, $\|\overline{\mathbf{e}}\|_{2}=\left\|\mathbf{T}^{-1} \mathbf{e}\right\|_{2} \leq$ $\left\|\mathbf{T}^{-1}\right\|_{2}\|\mathbf{e}\|_{2}$. Therefore,

$$
\mu<\|\overline{\mathbf{e}}\|_{2} \leq\left\|\mathbf{T}^{-1}\right\|_{2}\|\mathbf{e}\|_{2}
$$

that is, $\|\mathbf{e}\|_{2}>\mu_{0}, \mu_{0}=\delta / \lambda_{1}$. Here, $-\lambda_{1}$ is the maximum eigenvalue of matrix $\mathbf{A}$.

Define the maximum eigenvalue of matrix $\mathbf{A}$ is $\lambda_{\max }(\mathbf{A})$, and the minimum eigenvalue of matrix $\mathbf{A}$ is $\lambda_{\min }(\mathbf{A})$; then, $\lambda_{\max }(\mathbf{A})=-\lambda_{1}$ and $\lambda_{1}=-\lambda_{\max }(\mathbf{A})$.

Therefore, if $\|\mathbf{e}\|_{2}>\mu_{0}, \mu_{0}=-\delta /\left(\lambda_{\max }(\mathbf{A})\right)$, that is, $\lambda_{\max }(\mathbf{A})<-\delta /\|\mathbf{e}\|_{2}, \dot{V}(\mathbf{e})<0$.

Moreover, considering that $V(\mathbf{e})=(1 / 2) \mathbf{e}^{T} \mathbf{P e}$ and $(1 / 2) \lambda_{\min }(\mathbf{P})\|\mathbf{e}\|_{2}^{2} \leq(1 / 2) \mathbf{e}^{T} \mathbf{P e} \leq(1 / 2) \lambda_{\max }(\mathbf{P})\|\mathbf{e}\|_{2}^{2}$, let $\alpha_{1}(r)=$
$(1 / 2) \lambda_{\min }(\mathbf{P}) r^{2}$ and $\alpha_{2}(r)=(1 / 2) \lambda_{\max }(\mathbf{P}) r^{2}$, we have $\alpha_{1}^{-1}(r)=$ $\sqrt{2 r /\left(\lambda_{\min }(\mathbf{P})\right)}$

According to lemma, we have

$$
\begin{aligned}
\|\mathbf{e}\| \leq \alpha_{1}^{-1}\left(\alpha_{2}\left(\mu_{0}\right)\right) & =\sqrt{\frac{2 \alpha_{2}\left(\mu_{0}\right)}{\lambda_{\min }(\mathbf{P})}}=\sqrt{\frac{\lambda_{\max }(\mathbf{P}) \mu_{0}^{2}}{\lambda_{\min }(\mathbf{P})}} \\
& =-\frac{\delta}{\lambda_{\max }(\mathbf{A})} \sqrt{\frac{\lambda_{\max }(\mathbf{P})}{\lambda_{\min }(\mathbf{P})}}
\end{aligned}
$$

that is, $\|\mathbf{e}\|=O(1)$, and the upper bound of the tracking error is $-\left(\left(\delta /\left(\lambda_{\max }(\mathbf{A})\right)\right) \sqrt{\left(\lambda_{\max }(\mathbf{P})\right) /\left(\lambda_{\min }(\mathbf{P})\right)}\right)$. q.e.d.

3.3. Design Procedures. For the disturbance rejection control law (6), state observer (9), and disturbance observer (11), 


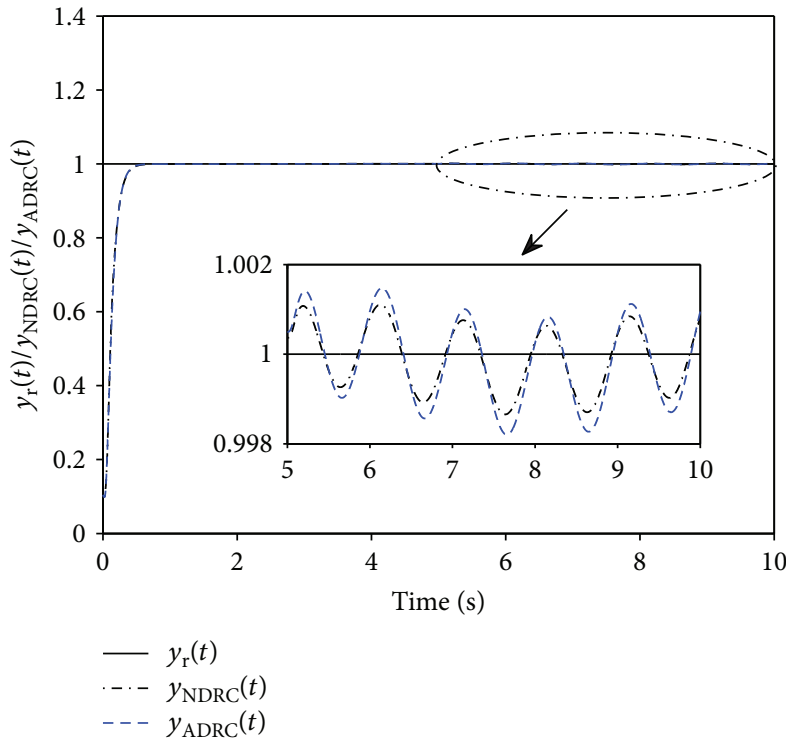

(a)

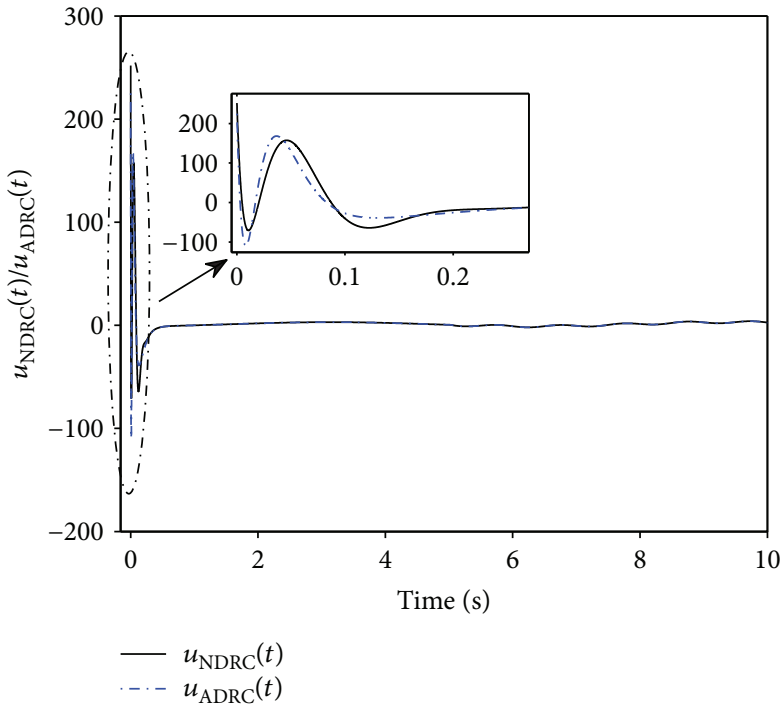

(b)

Figure 4: Comparisons between NDRC and ADRC.

TABLE 2: Comparisons of NDRC and ADRC for microcantilever in the AFM system.

\begin{tabular}{lcccc}
\hline Controller & $\begin{array}{c}\text { Simulation } \\
\text { time }(\mathrm{s})\end{array}$ & $\begin{array}{c}\text { Control } \\
\text { on }(\mathrm{s})\end{array}$ & $\begin{array}{c}\text { External } \\
\text { disturbance on }(\mathrm{s})\end{array}$ & ITAE \\
\hline NDRC & 10 & 0 & 5 & 0.0402 \\
ADRC & 10 & 0 & 5 & 0.0493 \\
\hline
\end{tabular}

parameters a, $\mathbf{L}, l_{n+1}$ have to be determined. Design procedures can be summarized as follows.

Step 1. Design the state observer according to (9). Let the eigenvalue of the state observer be

$$
\left|\lambda \mathbf{I}-\mathbf{A}_{G}\right|=\left|\lambda \mathbf{I}-\left(\mathbf{A}_{\mathbf{x}}-\mathbf{L C}\right)\right|=\left(\lambda+\omega_{o}\right)^{n},
$$

where $\omega_{o}$ is the bandwidth of the state observer. For the second-order system, we have $\mathbf{L}=\left[l_{1}, l_{2}\right]^{T}=\left[2 \omega_{o}, \omega_{o}^{2}\right]^{T}$, and for the third-order system, we have $\mathbf{L}=\left[l_{1}, l_{2}, l_{3}\right]^{T}=$ $\left[3 \omega_{o}, 3 \omega_{o}^{2}, \omega_{o}^{3}\right]^{T}$.

Step 2. Design a disturbance observer according to (11). Choose a proper gain $l_{n+1}$.

Step 3. Choosing system eigenvalues to be $-\lambda_{1},-\lambda_{2}, \ldots,-\lambda_{n}$, then we have $\overline{\mathbf{A}}=-\operatorname{diag}\left[\lambda_{1}, \lambda_{2}, \ldots, \lambda_{n}\right]$.

Step 4. According to Vandermonde matrix $\mathbf{T}$, we have $\mathbf{A}=\mathbf{T} \overline{\mathbf{A}} \mathbf{T}^{-1}$.

Step 5. The last row of matrix $\mathbf{A}=\mathbf{T} \overline{\mathbf{A}} \mathbf{T}^{-1}$ is the opposite number of the control parameter vector $\mathbf{a}=$ $\left[a_{n}, a_{n-1}, \ldots, a_{1}\right]^{T}$.
TABLE 3: Control parameters of NDRC and ADRC (ii).

\begin{tabular}{lcccccccc}
\hline Controller & $\omega_{\mathrm{o}}$ & $l_{3} / \omega_{c}$ & $\lambda_{1} / b_{0}$ & $\lambda_{2} / \beta_{1}$ & $a_{1} / \beta_{2}$ & $a_{2} / \beta_{3}$ & $k_{1}$ & $k_{2}$ \\
\hline NDRC & 120 & 36 & -10 & -9 & 19 & 90 & - & - \\
ADRC & 75 & 15 & 1 & 225 & 16,875 & 421,875 & 30 & 225
\end{tabular}

\section{Numerical Simulations}

In this section, three nonlinear systems are selected to confirm the new disturbance rejection control (NDRC) proposed in this paper. Cases in the absence and presence of external sinusoidal disturbance are considered. In all simulations, external disturbances $d(t)$ are set to be $\sin (2 \pi t)$.

In addition, NDRC and ADRC have been compared. Integral of time-multiplied absolute value of error (ITAE) values are listed to present the difference. Parameters of ADRC are chosen according to the bandwidth parameterization approach proposed by Gao [22].

Example 1. The dynamics of microcantilever in atomic force microscope (AFM) system is [23]

$$
\begin{aligned}
& \dot{x}_{1}=x_{2}, \\
& \dot{x}_{2}=-\delta x_{2}-x_{1}+F \cos (\Omega t)+F_{I L}\left(x_{1}(t)\right),
\end{aligned}
$$

where $t$ is the time, $x_{1}$ and $x_{2}$ are dimensionless position and velocity of the microcantilever tip, $F$ and $\Omega$ are amplitude and frequency of the forcing term, and $\delta$ is the damping factor. $F_{I L}\left(x_{1}(t)\right)=\left(\sigma^{6} d_{1} /\left(30\left(\alpha+x_{1}(t)\right)^{8}\right)\right)-$ $d_{1} /\left(\alpha+x_{1}(t)\right)^{2}$ denotes the attraction/repulsion interaction force derived from Lennard-Jones interaction potential.

System parameters are chosen to be $\delta=0.04, \sigma=$ $0.3, \alpha=0.8, F=2.0, \Omega=1, d_{1}=4 / 27$, and initial states 


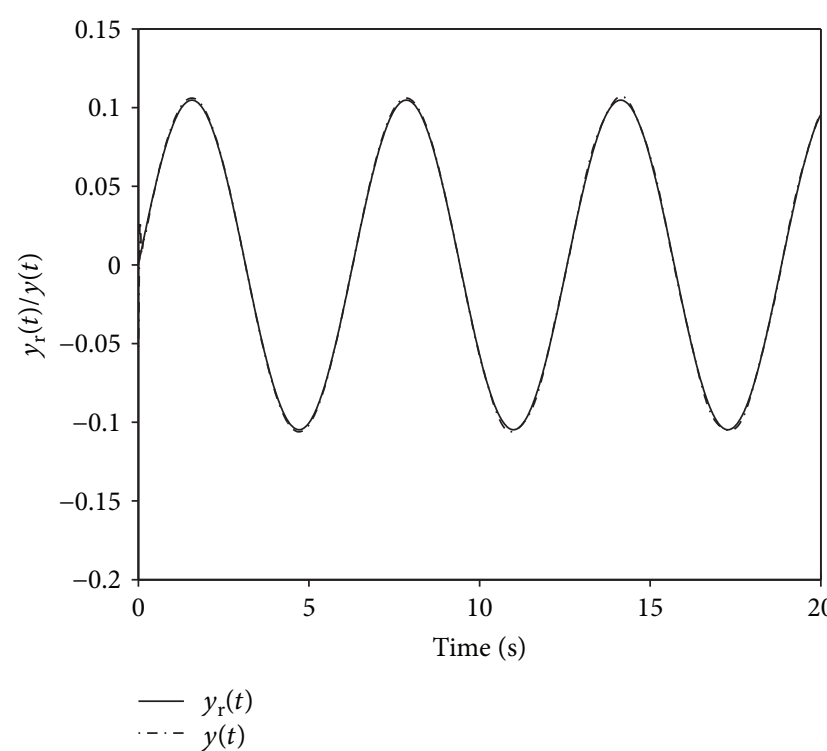

(a)

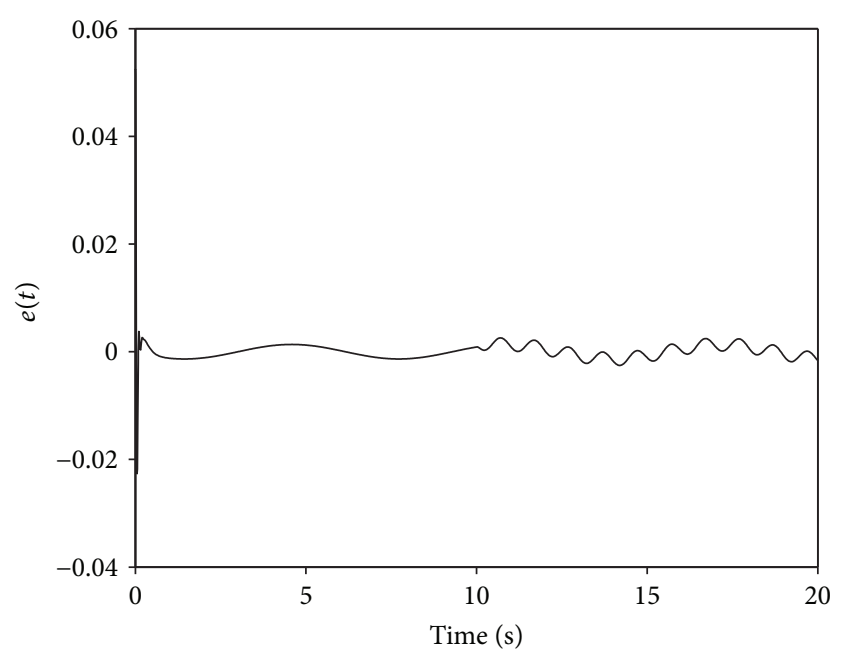

(c)

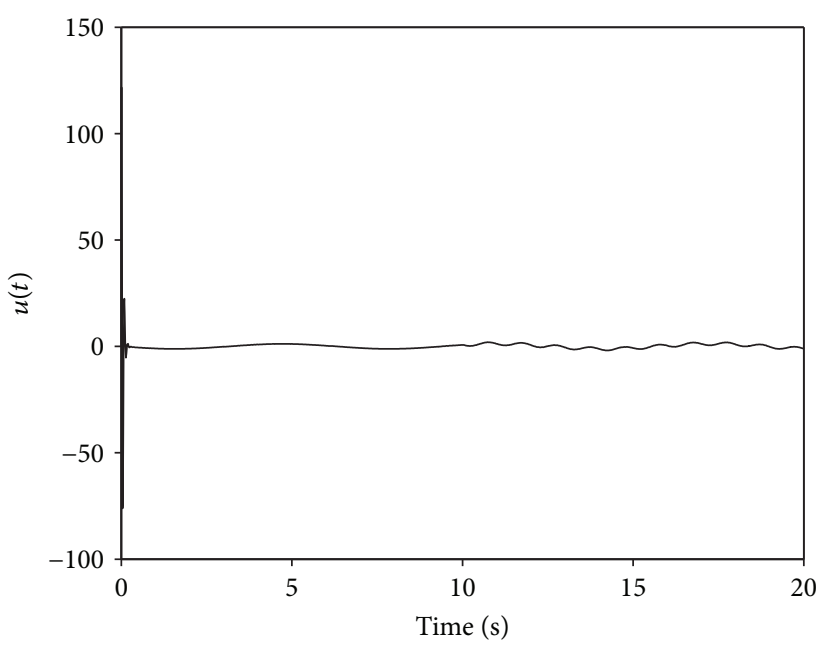

(b)

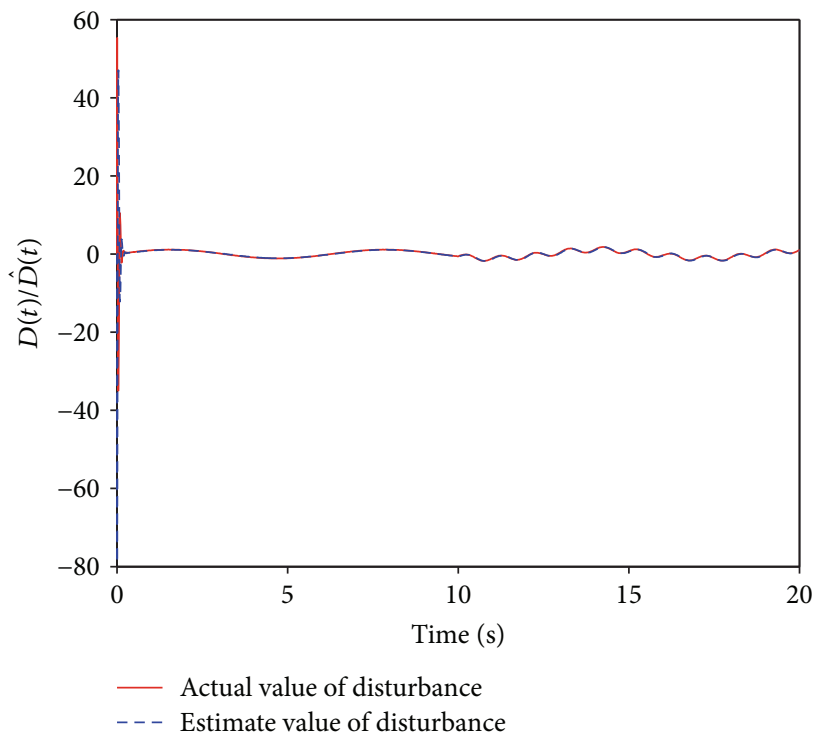

(d)

FIGURE 5: System response of inverted pendulum by NDRC.

$\left[x_{1}(0), x_{2}(0)\right]=[0.1,0.2]^{T}$. Chaotic dynamics behaviors and attractor of microcantilever in AFM are given in Figure 2.

In this simulation, we try to make the output of the chaotic dynamic system to track a fixed value. Simulation is performed for 10 seconds. Disturbance is introduced from the 5th second, and it lasts to the end of the simulation. Controller parameters are shown in Table 1 (see NDRC (i)). System responses are presented in Figure 3.

From Figure 3, we can see that NDRC is able to get satisfied performance even if there exists sinusoidal disturbance.

In order to make a comparison with active disturbance rejection control (ADRC), simulations have been performed. Numerical results are shown in Figure 4. Control parameters are also given in Table 1 (see NDRC (ii) and ADRC).
From Figure 4, we can see clearly that, when control signals are close, oscillation amplitudes of NDRC are smaller than those of ADRC in the presence of sinusoidal disturbance. It signifies that NDRC is superior to ADRC in suppressing sinusoidal disturbance. ITAE values shown in Table 2 also confirm the fact.

Example 2. The inverted pendulum system dynamics is [24]

$$
\begin{aligned}
\dot{x}_{1}= & x_{2}, \\
\dot{x}_{2}= & \frac{g \sin x_{1}-m l x_{2}^{2} \cos x_{1} \sin x_{1} /\left(m_{\mathrm{c}}+m\right)}{l\left(4 / 3-m \cos ^{2} x_{1} /\left(m_{\mathrm{c}}+m\right)\right)} \\
& +\frac{\cos x_{1} /\left(m_{\mathrm{c}}+m\right)}{l\left(4 / 3-m \cos ^{2} x_{1} /\left(m_{\mathrm{c}}+m\right)\right)} u, \\
y= & x_{1},
\end{aligned}
$$




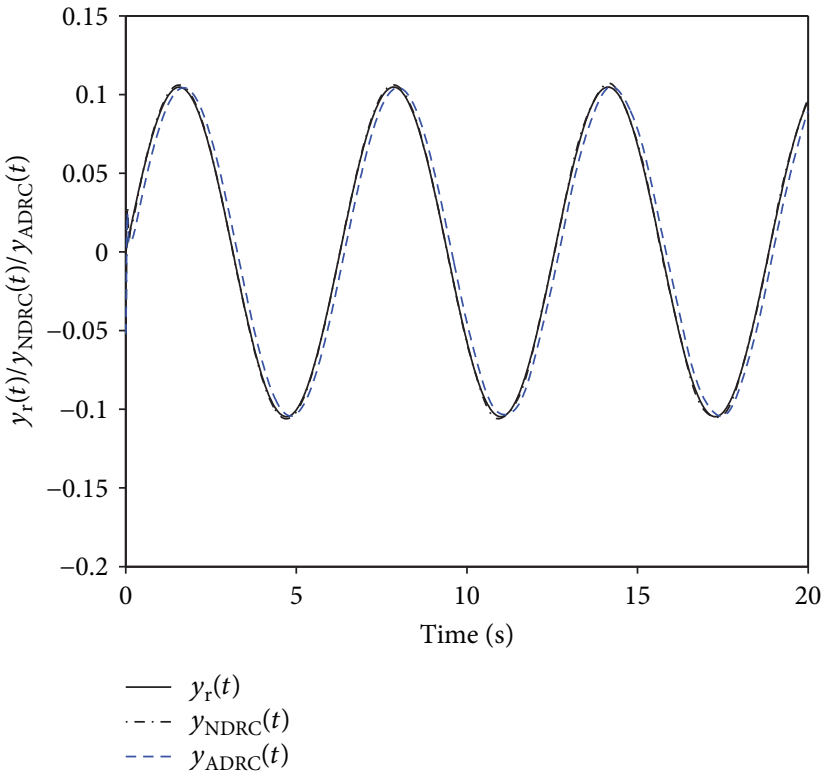

(a)

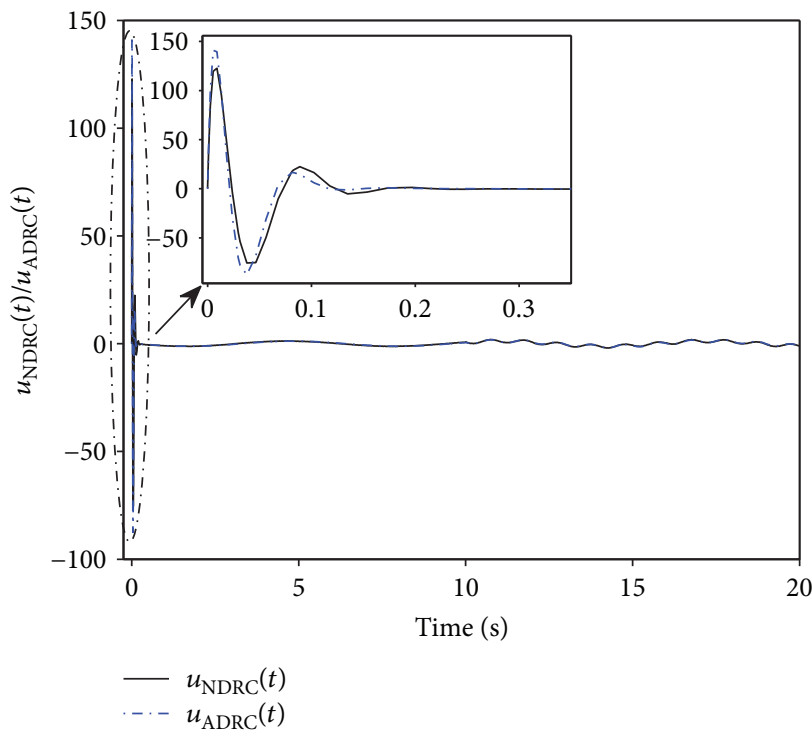

(b)

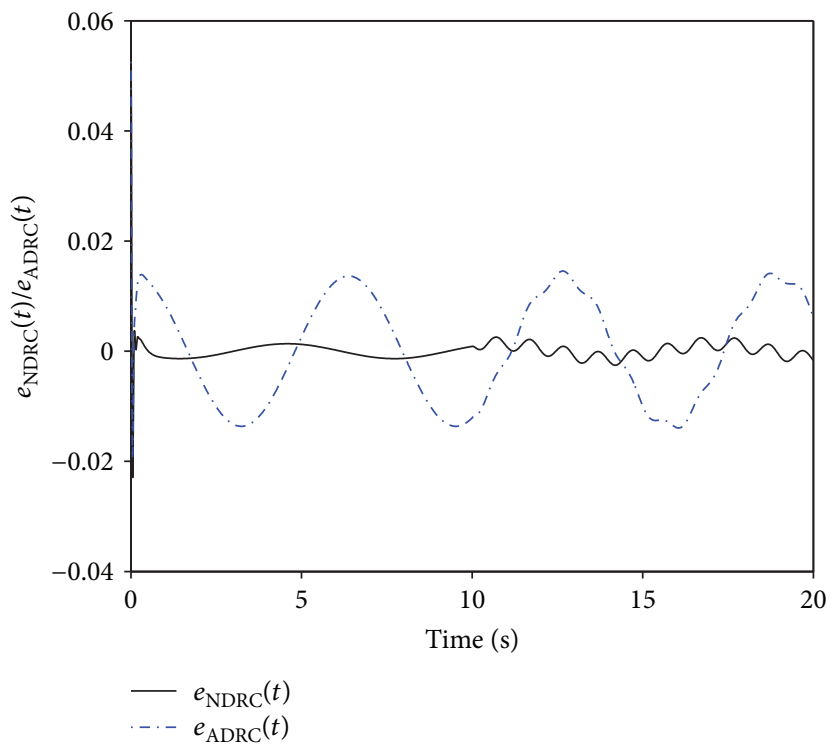

(c)

Figure 6: Comparisons between NDRC and ADRC.

TABle 4: Comparisons of NDRC and ADRC for the inverted pendulum system.

\begin{tabular}{lcccc}
\hline Controller & $\begin{array}{c}\text { Simulation } \\
\text { time }(\mathrm{s})\end{array}$ & $\begin{array}{c}\text { Control } \\
\text { on }(\mathrm{s})\end{array}$ & $\begin{array}{c}\text { External } \\
\text { disturbance on }(\mathrm{s})\end{array}$ & ITAE \\
\hline NDRC & 20 & 0 & 10 & 0.1933 \\
ADRC & 20 & 0 & 10 & 1.7943 \\
\hline
\end{tabular}

where $x_{1}, x_{2}$ are the angular position and velocity of the pole. $g=9.8 \mathrm{~m} / \mathrm{s}^{2}$ is the acceleration due to gravity, $m_{\mathrm{c}}=1 \mathrm{~kg}$ is the mass of the cart, $m=0.1 \mathrm{~kg}$ is the mass of the pole, $l=0.5 \mathrm{~m}$ is the half-length of the pole, and $u$ is the applied force. Our objective is to maintain the system output to track the desired trajectory $\mathbf{y}_{r}=\pi / 30 \sin (t)$. The initial states are chosen to be $[-\pi / 60,0]^{T}$. Controller parameters are listed in Table 3.

Simulation results are shown in Figure 5.

Figure 5 shows that NDRC is also capable of tracking sinusoidal signal in the presence of sinusoidal disturbance.

Comparisons between NDRC and ADRC have also been performed. Parameters of NDRC and ADRC are taken in which the values are given in Table 3. Simulation results are presented in Figure 6. ITAE values are listed in Table 4. 


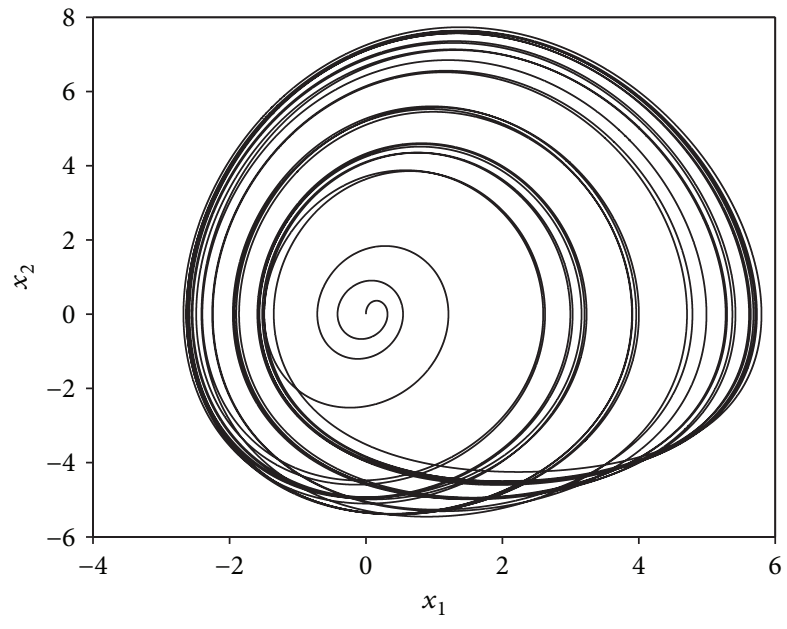

(a)

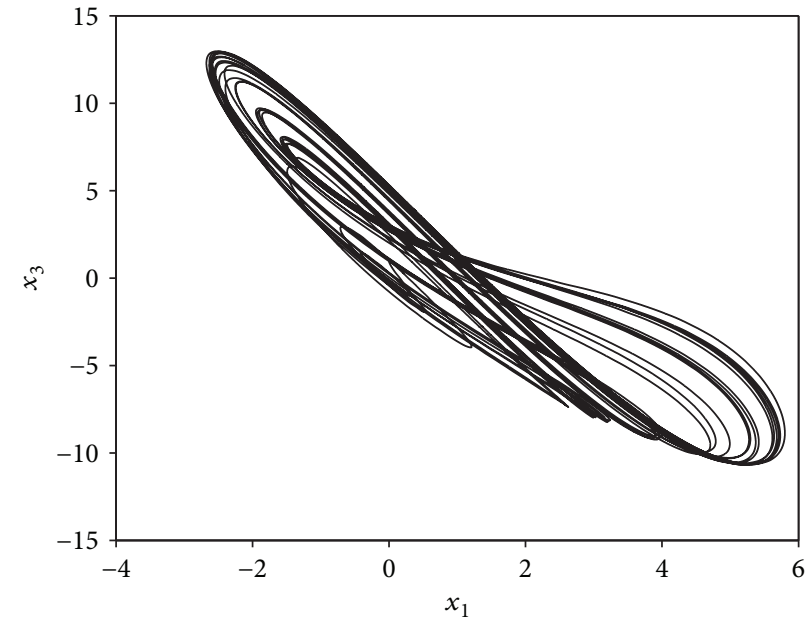

(b)

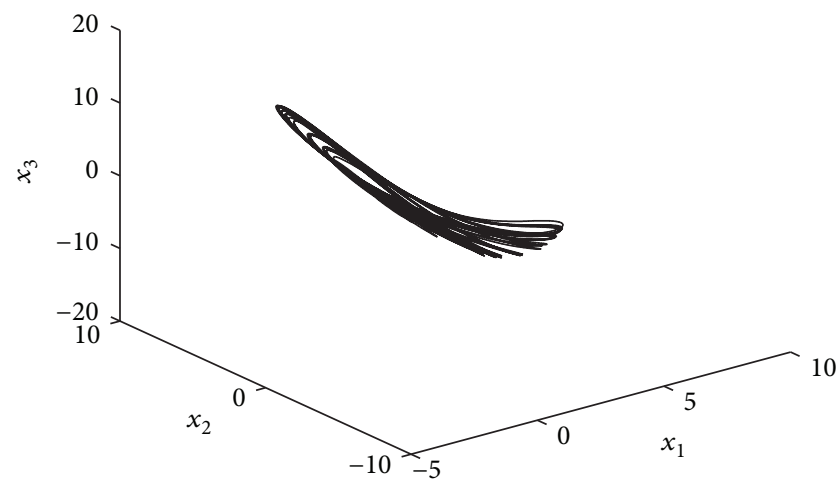

(c)

Figure 7: Chaotic attractor of Genesio-Tesi system.

TABLE 5: Control parameters of NDRC and ADRC (iii).

\begin{tabular}{lcccccccccc}
\hline Controller & $\omega_{0}$ & $l_{4} / \omega_{c}$ & $\lambda_{1} / b_{0}$ & $\lambda_{2} / \beta_{1}$ & $\lambda_{3} / \beta_{2}$ & $a_{1} / \beta_{3}$ & $a_{2} / \beta_{4}$ & $a_{3} / k_{1}$ & $k_{2}$ & $k_{3}$ \\
\hline NDRC & 120 & 36 & -10 & -9 & -8 & 27 & 242 & 720 & - & - \\
ADRC & 45 & 9 & 1 & 180 & 12,150 & 364,500 & $4,100,625$ & 729 & 243 & 27 \\
\hline
\end{tabular}

Figure 6 shows that with less control energy (see Figure 6(b)), NDRC is able to track the sinusoidal signal with no phase delay (see Figure 6(a)). Figure 6(c) also depicts the fact vividly. Additionally, when sinusoidal disturbance is introduced, NDRC can achieve much less tracking error, which means that NDRC is more effective in estimating and rejecting sinusoidal disturbance. ITAE values given in Table 4 show that the value of NDRC is improved by $89.23 \%$. It also verifies the disturbance estimation and rejection ability of NDRC.

Example 3. The uncertain Genesio-Tesi chaotic system can be written as [25]

$$
\begin{aligned}
& \dot{x}_{1}=x_{2}, \\
& \dot{x}_{2}=x_{3},
\end{aligned}
$$

$$
\begin{aligned}
\dot{x}_{3} & =-c x_{1}-b x_{2}-a x_{3}+m x_{1}^{2}+\Delta f(\mathbf{x}, t)+d(t)+u(t), \\
y & =x_{1},
\end{aligned}
$$

where $\mathbf{x}=\left[x_{1}, x_{2}, x_{3}\right]^{T} \in \mathbf{R}^{3}$ is the system state vector, constants $a, b, c, m$ are positive, $\Delta f(\mathbf{x}, t)$ is a time-varying function representing not precisely known and uncertain dynamics of chaotic systems, $d(t)$ is the external disturbance, and $u(t)$ is the control input.

In simulations, $a=1.2, b=2.92, c=6, m=1, \Delta f(\mathbf{x}, t)=$ $0.5 \sin \left(\pi x_{1}\right) \sin \left(2 \pi x_{2}\right) \sin \left(3 \pi x_{3}\right), d(t)=\sin (2 \pi t)$, and initial states are chosen to be $\left[x_{1}(0), x_{2}(0), x_{3}(0)\right]^{T}=[0,0,1]^{T}$. The chaotic attractor is shown in Figure 7.

In this case, we also drive system output to track a fixed value. Parameters of chaos control are given in Table 5. 


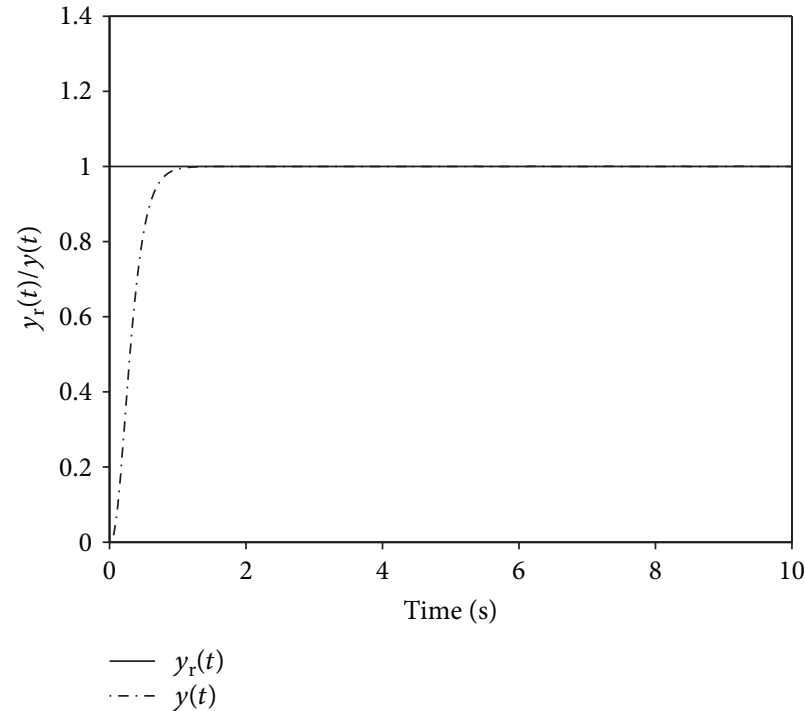

(a)

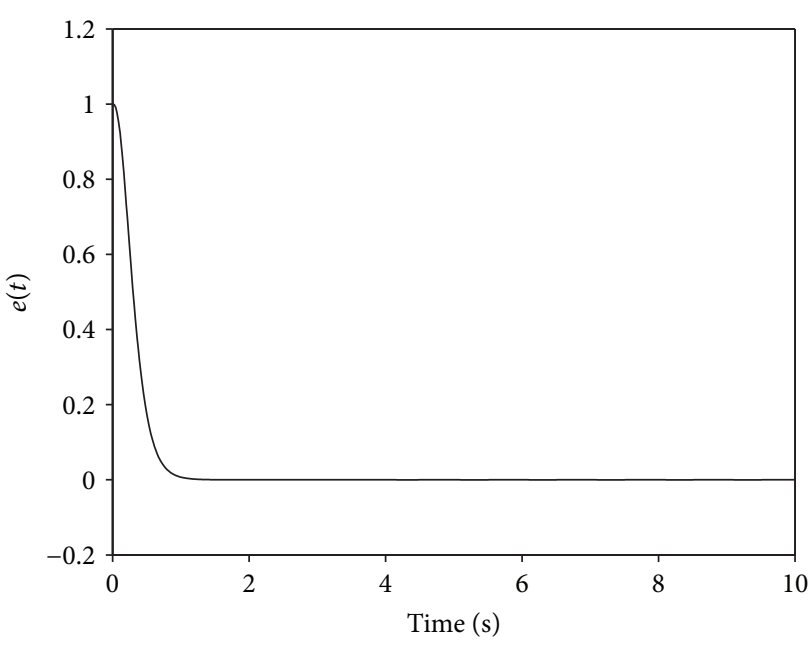

(c)

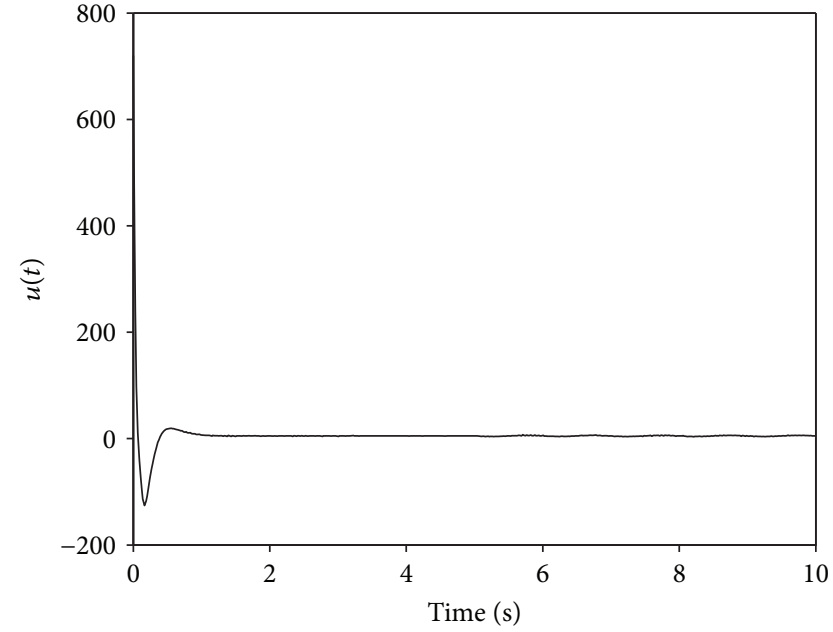

(b)

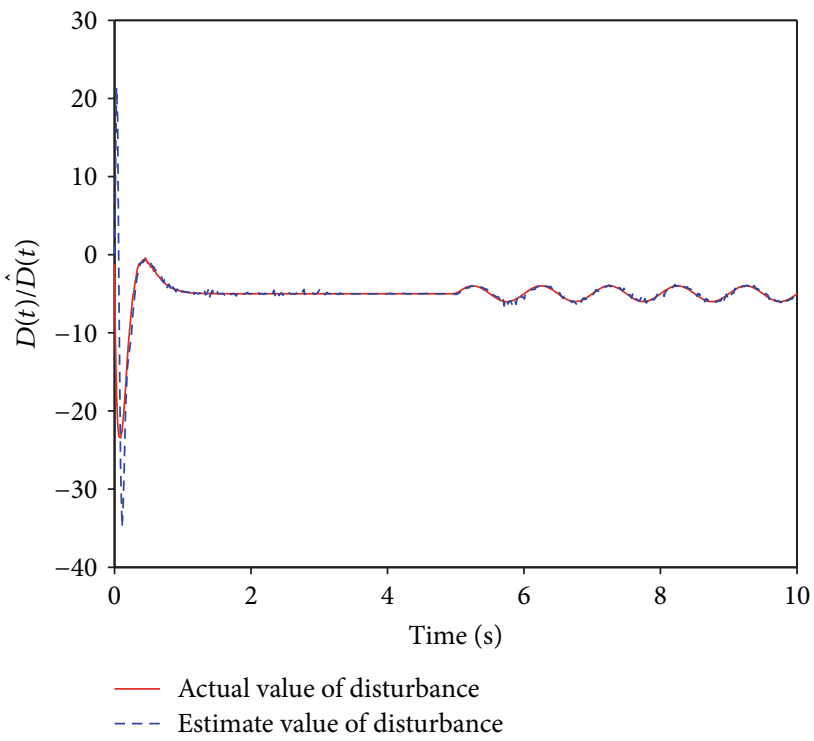

(d)

Figure 8: System response of the Genesio-Tesi system by NDRC.

System response can be found in Figure 8.

Figure 8 shows that, with the help of disturbance observer, NDRC is able to track desired trajectory regardless if sinusoidal disturbance exists or not. Comparisons between NDRC and ADRC have been performed; Figure 9 and Table 6 give out the difference.

From Figure 9, we can see that both NDRC and ADRC are capable of estimating and compensating disturbance to guarantee system performance. However, in the presence of sinusoidal disturbance, NDRC is able to provide much smaller oscillation amplitudes with similar control energy. ITAE values shown in Table 6 also confirm that NDRC is more effective in estimating and cancelling uncertainties and disturbances.

\section{Conclusion and Outlook}

Driven by practical engineering needs, disturbance attenuation/rejection control methods have been developed in various industrial sectors. In this paper, a new disturbance rejection control algorithm has also been put forward to realize the control of nonlinear systems with uncertainties. With the help of a disturbance observer and a baseline controller, nonlinear systems can be dynamically linearized and system dynamics is approximate to a LTI system with controllable canonical form. Furthermore, based on the results obtained, any effective control algorithms, which are suitable for controllable canonical form, are also able to be utilized in the disturbance rejection control scheme 


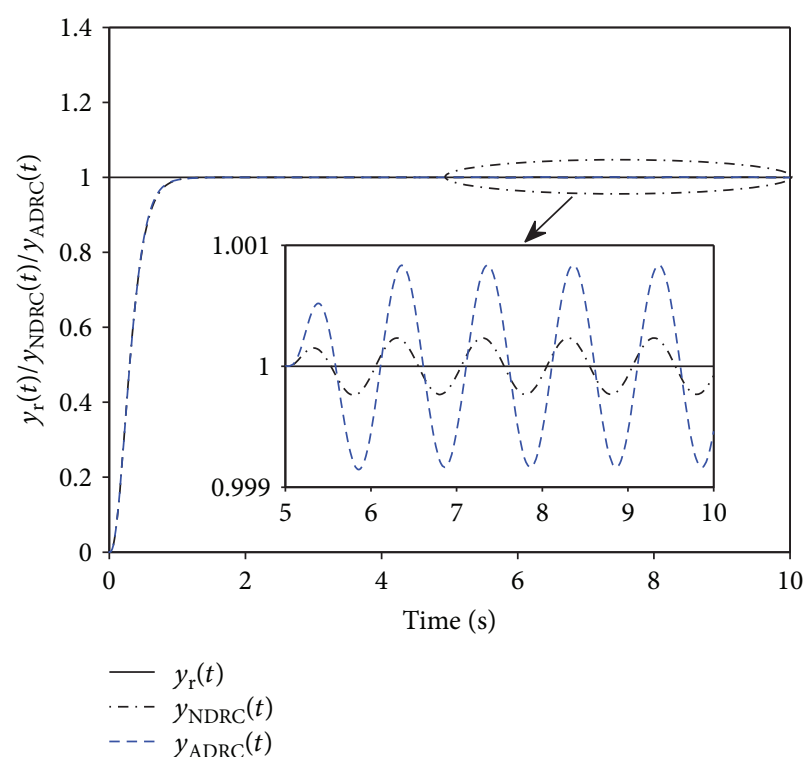

(a)

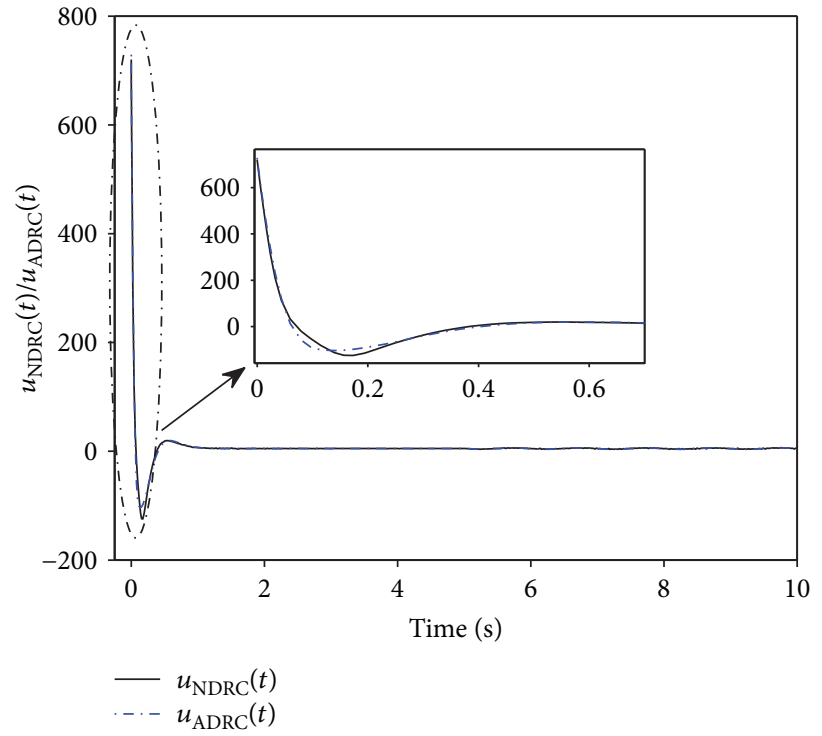

(b)

Figure 9: Comparisons between NDRC and ADRC.

TABle 6: Comparisons of NDRC and ADRC for the Genesio-Tesi chaotic system.

\begin{tabular}{lcccc}
\hline Controller & $\begin{array}{c}\text { Simulation } \\
\text { time }(\mathrm{s})\end{array}$ & $\begin{array}{c}\text { Control } \\
\text { on }(\mathrm{s})\end{array}$ & $\begin{array}{c}\text { External disturbance } \\
\text { on }(\mathrm{s})\end{array}$ & ITAE \\
\hline NDRC & 10 & 0 & 5 & 0.0810 \\
ADRC & 10 & 0 & 5 & 0.0939 \\
\hline
\end{tabular}

proposed in this paper. In addition, although numerical simulation results are presented, the experimental results are also on the way.

\section{Data Availability}

The data used to support the findings of this study are available from the corresponding author upon request.

\section{Conflicts of Interest}

The authors declare that there is no conflict of interest regarding the publication of this paper.

\section{Acknowledgments}

This work is supported by the National Natural Science Foundation of China (61403006), Key Program of Beijing Municipal Education Commission (KZ201810011012), and project of high-level teachers in Beijing municipal universities in the period of the 13th Five-Year Plan (CIT\&TCD201704044).

\section{References}

[1] Š. Kozák, "State-of-the-art in control engineering," Journal of Electrical Systems and Information Technology, vol. 1, no. 1, pp. 1-9, 2014.
[2] Z. Gao, "On the centrality of disturbance rejection in automatic control," ISA Transactions, vol. 53, no. 4, pp. 850857, 2014

[3] W.-H. Chen, J. Yang, L. Guo, and S. Li, "Disturbanceobserver-based control and related methods-an overview," IEEE Transactions on Industrial Electronics, vol. 63, no. 2, pp. 1083-1095, 2016.

[4] K. Ohishi, M. Nakao, K. Ohnishi, and K. Miyachi, "Microprocessor-controlled DC motor for load-insensitive position servo system," IEEE Transactions on Industrial Electronics, vol. IE-34, no. 1, pp. 44-49, 1987.

[5] W.-H. Chen, D. J. Ballance, P. J. Gawthrop, and J. O’Reilly, "A nonlinear disturbance observer for robotic manipulators," IEEE Transactions on Industrial Electronics, vol. 47, no. 4, pp. 932-938, 2000.

[6] D. Ginoya, P. D. Shendge, and S. B. Phadke, "Disturbance observer based sliding mode control of nonlinear mismatched uncertain systems," Communications in Nonlinear Science and Numerical Simulation, vol. 26, no. 1-3, pp. 98-107, 2015.

[7] C. Johnson, "Accomodation of external disturbances in linear regulator and servomechanism problems," IEEE Transactions on Automatic Control, vol. 16, no. 6, pp. 635-644, 1971.

[8] J. Han, "From PID to active disturbance rejection control," IEEE Transactions on Industrial Electronics, vol. 56, no. 3, pp. 900-906, 2009.

[9] S. J. Kwon and W. K. Chung, "A discrete-time design and analysis of perturbation observer for motion control applications," IEEE Transactions on Control Systems Technology, vol. 11, no. 3, pp. 399-407, 2003.

[10] H. Sira-Ramírez, J. Linares-Flores, C. García-Rodríguez, and M. A. Contreras-Ordaz, "On the control of the permanent magnet synchronous motor: an active disturbance rejection control approach," IEEE Transactions on Control Systems Technology, vol. 22, no. 5, pp. 20562063, 2014. 
[11] A. Radke and Z. Gao, "A survey of state and disturbance observers for practitioners," in 2006 American Control Conference, pp. 5183-5188, Minneapolis, MN, USA, June 2006.

[12] L. Guo and S. Cao, "Anti-disturbance control theory for systems with multiple disturbances: a survey," ISA Transactions, vol. 53, no. 4, pp. 846-849, 2014.

[13] K. Ohishi, K. Ohnishi, and K. Miyachi, "Torque-speed regulation of $\mathrm{dc}$ motor based on load torque estimation method," in IEEJ International Power Electronics Conference, pp. 1209-1218, IPEC-TOKYO, 1983.

[14] J. Han, "Extended state observer for a class of uncertain plants," Control and Decision, vol. 10, no. 1, pp. 85-88, 1995.

[15] L. Guo and W.-H. Chen, "Disturbance attenuation and rejection for systems with nonlinearity via DOBC approach," International Journal of Robust and Nonlinear Control, vol. 15, no. 3, pp. 109-125, 2005.

[16] L. Guo and S. Cao, Anti-Disturbance Control for Systems with Multiple Disturbances, CRC Press, London, U.K., 2013.

[17] J. Yang, H. Cui, S. Li, and A. Zolotas, "Optimized active disturbance rejection control for DC-DC buck converters with uncertainties using a reduced-order GPI observer," IEEE Transactions on Circuits and Systems I: Regular Papers, vol. 65, no. 2, pp. 832-841, 2018.

[18] Y. Jiang, Q. Sun, X. Zhang, and Z. Chen, "Pressure regulation for oxygen mask based on active disturbance rejection control," IEEE Transactions on Industrial Electronics, vol. 64, no. 8, pp. 6402-6411, 2017.

[19] J. Na, A. S. Chen, G. Herrmann, R. Burke, and C. Brace, "Vehicle engine torque estimation via unknown input observer and adaptive parameter estimation," IEEE Transactions on Vehicular Technology, vol. 67, no. 1, pp. 409-422, 2018.

[20] S. Li and J. Li, "Output predictor-based active disturbance rejection control for a wind energy conversion system with PMSG," IEEE Access, vol. 5, no. 99, pp. 5205-5214, 2017.

[21] H. Khalil, Nonlinear Systems, Prentice Hall, Third edition, 2002.

[22] Z. Gao, "Scaling and bandwidth-parameterization based controller tuning," in Proceedings of the 2003 American Control Conference, 2003, pp. 4989-4996, Denver, CO, USA, June 2003.

[23] M. T. Arjmand, H. Sadeghian, H. Salarieh, and A. Alasty, "Chaos control in AFM systems using nonlinear delayed feedback via sliding mode control," Nonlinear Analysis: Hybrid Systems, vol. 2, no. 3, pp. 993-1001, 2008.

[24] H. F. Ho, Y. K. Wong, and A. B. Rad, "Adaptive fuzzy approach for a class of uncertain nonlinear systems in strictfeedback form," ISA Transactions, vol. 47, no. 3, pp. 286299, 2008.

[25] S. Dadras and H. R. Momeni, "Control uncertain Genesio-Tesi chaotic system: adaptive sliding mode approach," Chaos, Solitons and Fractals, vol. 42, no. 5, pp. 3140-3146, 2009. 


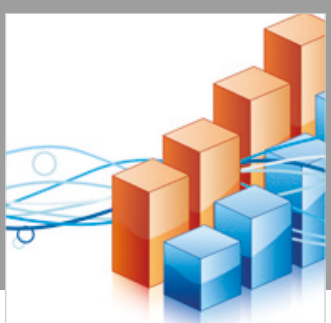

Advances in

Operations Research

\section{-n-m}
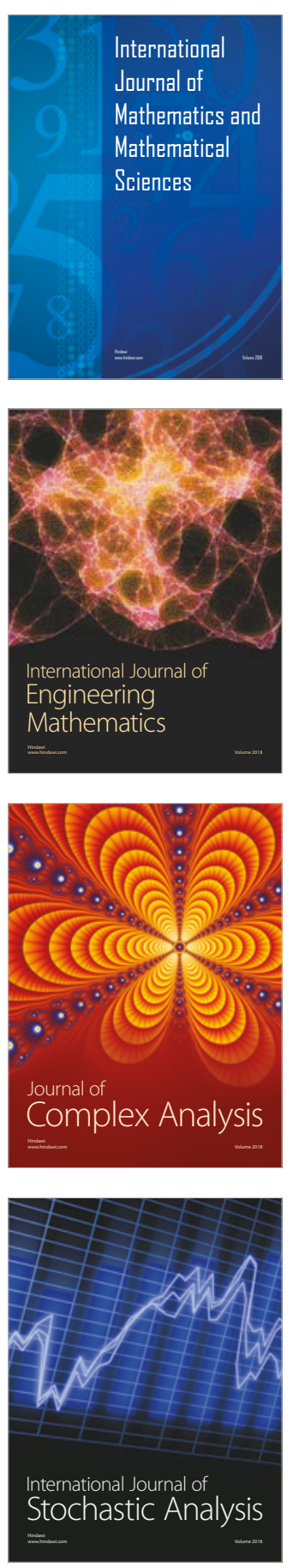
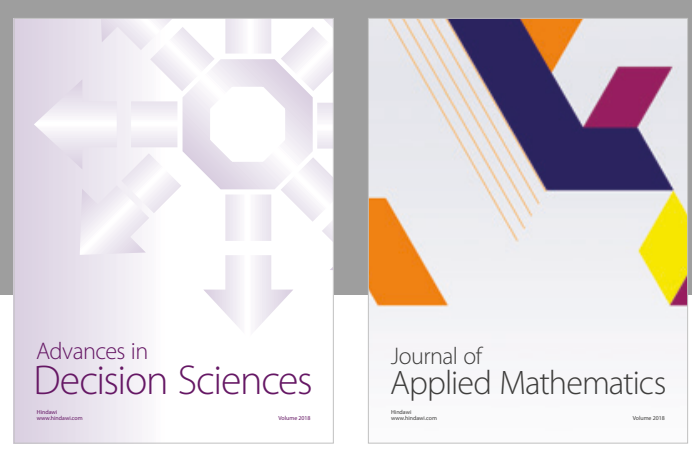

Journal of

Applied Mathematics
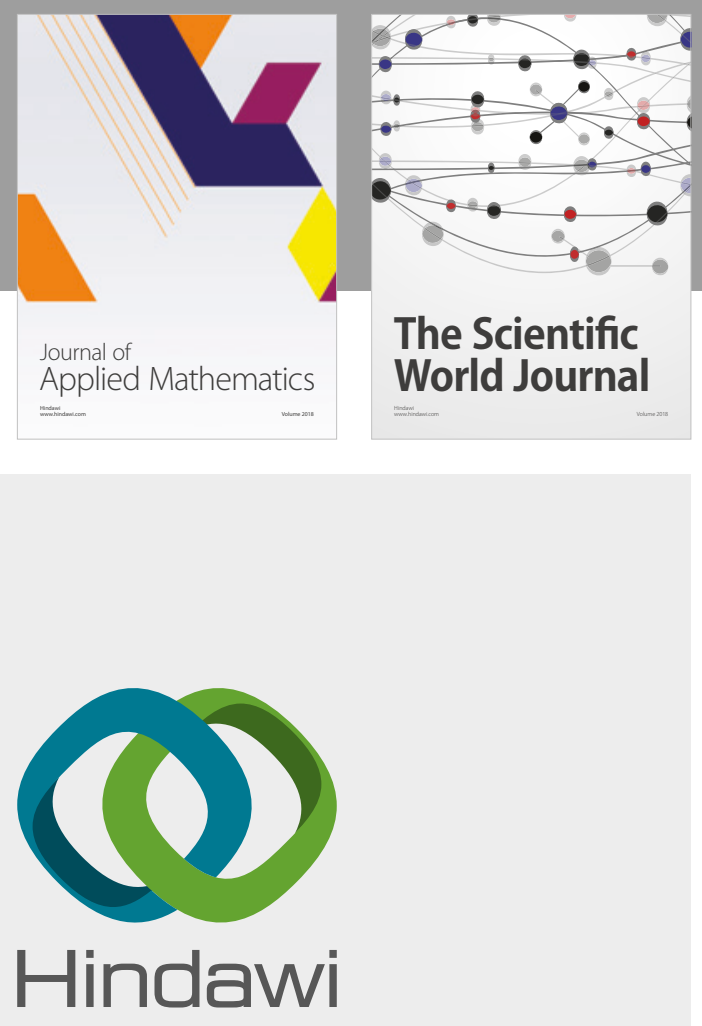

Submit your manuscripts at

www.hindawi.com

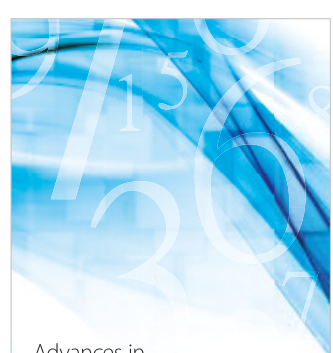

Advances in
Numerical Analysis
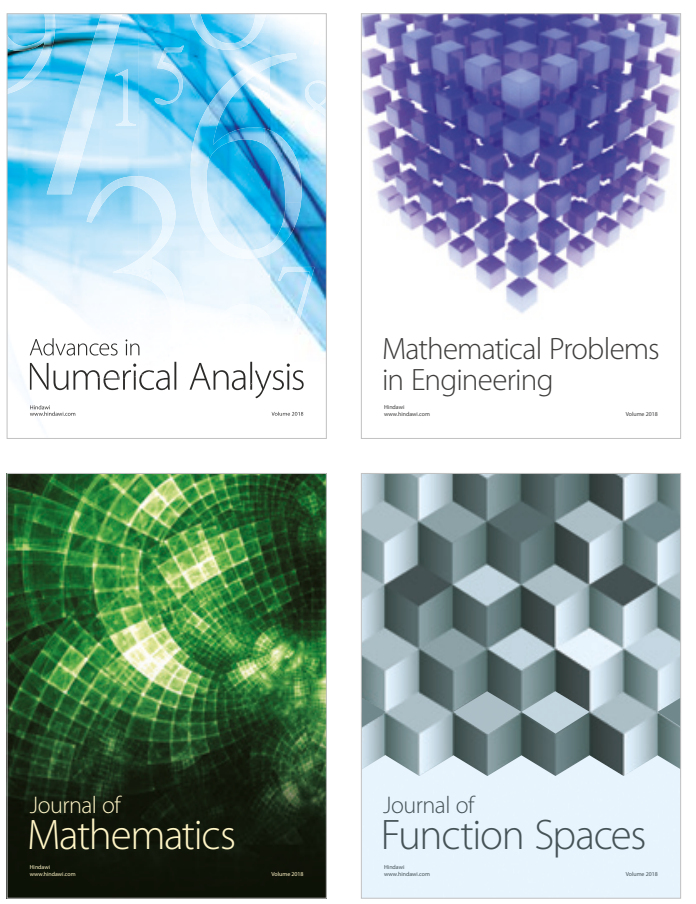

Mathematical Problems in Engineering

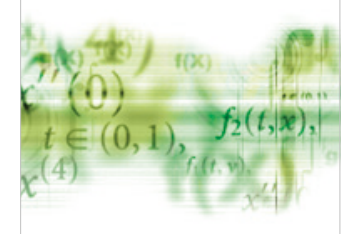

International Journal of

Differential Equations

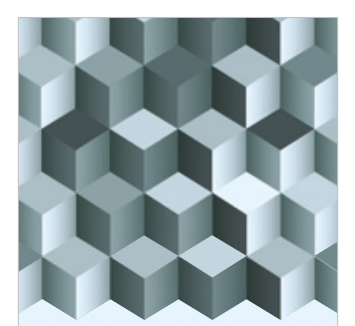

Journal of

Function Spaces
The Scientific

World Journal

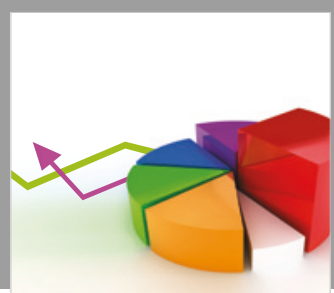

Journal of

Probability and Statistics
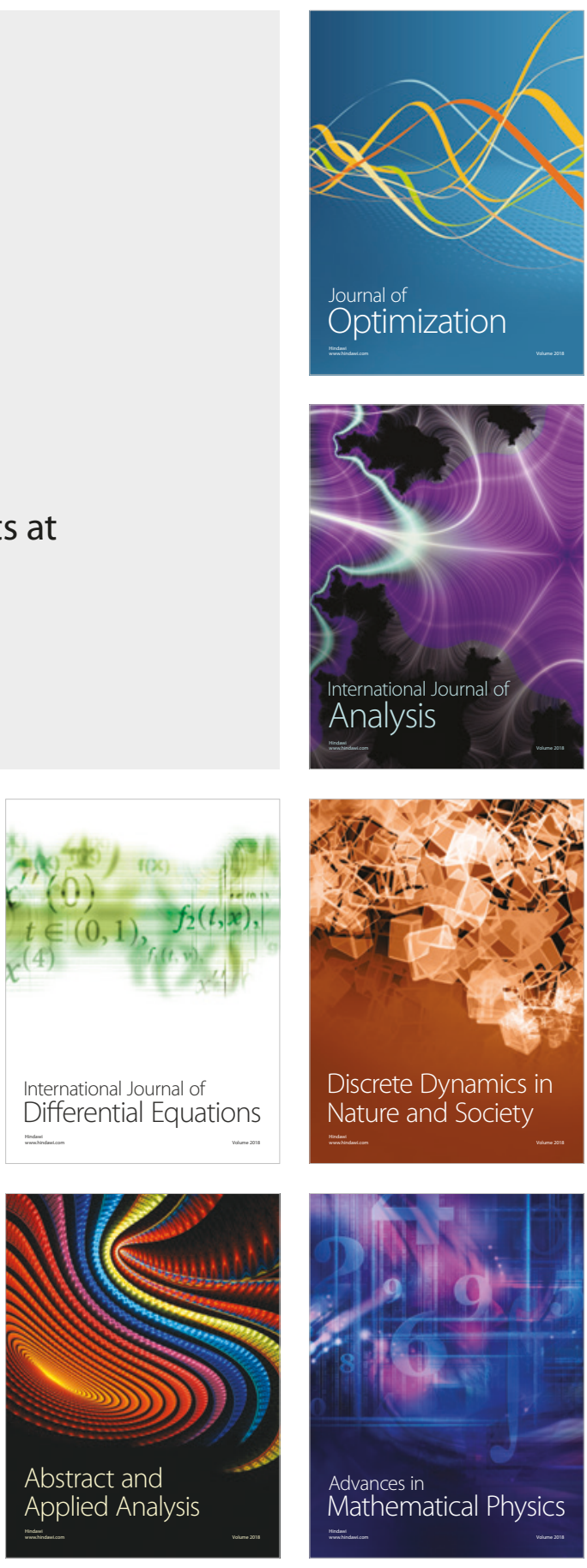